\title{
Strategies for Drug Encapsulation and Controlled Delivery Based on Vapor-Phase Deposited Thin Films
}

\author{
Alberto Perrotta, Oliver Werzer, and Anna Maria Coclite**
}

Vapor-phase deposition methods allow the synthesis and engineering of organic and inorganic thin films, with high control on the chemical composition, physical properties, and conformality. In this review, the recent applications of vapor-phase deposition methods such as initiated chemical vapor deposition (iCVD), plasma enhanced chemical vapor deposition (PE-CVD), and atomic layer deposition (ALD), for the encapsulation of active pharmaceutical drugs are reported. The strategies and emergent routes for the application of vapor-deposited thin films on the drug controlled release and for the engineering of advanced release nanostructured devices are presented.

preparation of solid state formulations like tablets, ${ }^{[17]}$ pellets, ${ }^{[18]}$ or powders. Developments in nanomaterial chemistry have enabled the proliferation of different types of drug carriers, for example, polymer-drug conjugates, nanoparticles, nanoclusters, micelles, dendritic polymer carriers, nanocapsules, and nanoemulsions. ${ }^{[19,20]}$ These can work as scaffolds in which the drug is loaded and preserved until delivery. The delivery can be spatio-temporally controlled to minimize side effects and maximize, instead, the therapeutic efficacy. ${ }^{[21]}$ Recently also metal-organic frameworks (MOF) (called also coordination polymers) have shown interesting results as drug carriers. ${ }^{[22,23]}$

\section{Introduction}

Drug therapies require the active pharmaceutical ingredients (API) to reach the desired site of action and the success of the treatment is governed by the time the drug requires to reach the target, the amount of drug assembling at the target site as well as its residence time. Furthermore, considerations like administration route, ${ }^{[1-3]}$ processing, ${ }^{[4,5]}$ and storage of drug formulations ${ }^{[5,6]}$ need attention as only the combination of all these allows the achievement of successful medications ready for therapy, marketing, and help for patients. Very often, drug molecules well perform only when assistance from other substances is provided. These substances (i.e., excipients) limit processing issues, ${ }^{[7]}$ mechanical and chemical instabilities, ${ }^{[8]}$ dissolution problems, ${ }^{[9]}$ resorption problems, ${ }^{[10]}$ and poor bio-availability or assist for better systemic circulation. ${ }^{[11]}$ Hence, the development of new therapeutic options is not limited to the synthesis and screening of new APIs, but also different administration routes and alternative dosage forms are extensively investigated. Generally, the latter is achieved by the engineering and processing of new excipients.

Ever since medications were used, excipients are employed. ${ }^{[2,13]}$ Especially, the usage of macromolecules or polymers ${ }^{[14-16]}$ as fillers, gliding agent, disintegration agent, or protective coatings allow the

Dr. A. M. Coclite, Dr. A. Perrotta

Institute of Solid State Physics, NAWI Graz, Graz University of Technology, 8010 Graz, Austria

E-mail: anna.coclite@tugraz.at

Dr. O. Werzer

Institute of Pharmaceutical Science, NAWI Graz, Department of Pharmaceutical Technology, University of Graz, 8010 Graz, Austria

The ORCID identification number(s) for the author(s) of this article can be found under https://doi.org/10.1002/adem.201700639.

DOI: 10.1002/adem.201700639
MOFs have very large and highly controlled porosity that can be used for loading and releasing the drug molecules. Solutions, dispersions, or emulsions of nanostructured materials conjugated with drugs hardly perform without large molecular weight species which provide, amongst others, solubility enhancement, ${ }^{[24]}$ viscosity adjustment, and stability. ${ }^{[25]}$ Another drawback of encapsulating drugs into nanocarriers is that only limited loads of medicine can be hosted, therefore multiple injections are required during a treatment cycle to deliver an optimal dose of drug to the desired site.

Alternatively, hydrogels and more generally polymers can offer interesting solutions that are more effective for long-term treatments. ${ }^{[26,27]}$ In this perspective, natural derived products like cellulose derivatives or gums ${ }^{[28]}$ are well established and approved to be used in medications. ${ }^{[25]}$ However, with progress in polymer chemistry, more synthetic counterparts with well-defined structures, narrow molecular weight distributions, and tunable properties are ready to be commercialized, enabling even more sophisticated medications. ${ }^{[29]}$ In this regard, polymers enable drug formulation functions like protection against premature release, and the stabilization of specific solid state forms. Furthermore, their potential for realization of site-specific drug release and activity are of paramount importance. ${ }^{[30-33]}$ Drug-loaded hydrogels can be directly injected in a minimally invasive way, thanks to their high moldability, and the ability to form tissue constructs in situ. ${ }^{[34]}$ Particularly interesting is the use of external stimuli to trigger the release of the drug at a specific site or specific time. ${ }^{[35-37]}$

\subsection{Polymers as API Carriers: Strategies and Diffusion Mechanisms}

To date, only a limited number of products are available which make use of a pure polymer to achieve therapeutic actions ${ }^{[38,39]}$; examples of such polymer therapeutics are Copaxone ${ }^{\circledR}$, 


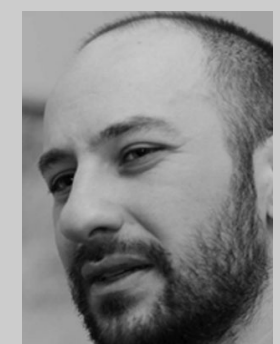

Alberto Perrotta obtained his Master in Chemical Science in 2012, at the University of Bari, Italy. He obtained his PhD at the Technical University of Eindhoven, the Netherlands, in 2016. He is currently a postdoctorate fellow at the Technical University of Graz, under the supervision of Dr. Anna Maria Coclite. His research focuses on thin film deposition and process development via (plasmabased) vapor-phase methods, porosity determination, and advanced opto-chemical characterization of thin films.

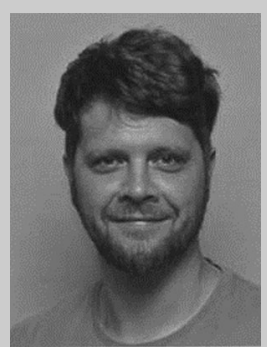

Oliver Werzer received a Master in Technical Physics in 2004 and his doctoral degree in 2008, both from the Graz University of Technology. After a Post-doc in the Chemistry department at the University of Newcastle (NSW, Australia), he joined the Department of Pharmaceutical Technology, (Institute of Pharmaceutical sciences, University of Graz, Austria). There, his team has been aiming to generate new pathways for manipulating the solid state properties of drugs by facilitating drug interface interactions.

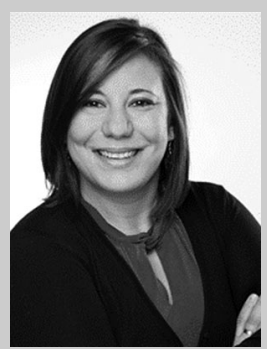

Anna Maria Coclite is Assistant Professor in the Institute of Solid State Physics of the Graz University of Technology. She was postdoctoral associate at the Massachusetts Institute of Technology, Department of Chemical Engineering, and received her PhD and M.S. degrees in Chemistry with the highest honors from the University of Bari, Italy in 2010 and 2006, respectively. Her current research interests include thin films technologies, nanomaterials, and surface modification through Chemical Vapor Deposition.

Hyaluronic acid, and Renagel ${ }^{\mathbb{B}}$. More common are instead drugpolymer conjugates, which can host other molecular species within the polymer backbone, ${ }^{[40,41]}$ either physisorbed or covalently bonded. For liquid drug formulations or fabrication processes, polymers are also used to adjust specific properties like viscosity, or enhance solubility, or stability. In addition, polymers also assist in fabricating more sophisticated or even smart drug release forms. ${ }^{[42-46]}$ As an example, having polymers of amphiphilic character, a polar and an apolar phase can be kept separated, so that the drug is encapsulated within the apolar phase. Such polymeric vehicles, similar to small molecules counterparts made from lipids or others, often provide enhanced drug stability. ${ }^{[45]}$ More importantly, such vehicles provide eventually very defined drug release capabilities, either due to controlled diffusion or triggered by external stimuli like $\mathrm{pH}$ and temperature change. ${ }^{[42-44,47,48]}$

When drug formulations are obtained from powders, they are generally addressed as solid state forms, and include tablets or pellets. Mostly, these are fabricated directly by compressing powder mixtures of an API and a polymer(s) or employing granulation routes. The high temperatures or pressures adopted in their processing induce polymer melting, which allows the production of solid state dispersions ${ }^{[49]}$ or solid state solutions applicable in standard extrusion ${ }^{[50]}$ or injection molding processes. ${ }^{[51]}$

In any case, the API release from a drug - polymer composite or matrix formulations results from either degradation/erosion/ disintegration of the polymer host or by molecular diffusion through the polymer host. For diffusion-limited drug release, matrix-, reservoir-, and hydrogel-based systems are usually differentiated. When the API is homogenously dispersed inside a suitable host, made from materials such as cellulose or lipids, the tortuosity of the matrix determines the drug release kinetics. Hydrogel drug formulations ${ }^{[2,53]}$ are similar to matrix systems as they are usually loaded with the API, but the drug release mechanism depends on swelling of the polymeric material and, thus, it is governed by the matrix mesh size. Furthermore, a reservoir-drug formulation is usually comprised of a core-shell structure. In such, the API is encapsulated and so protected from the dissolution medium by a defined membrane which, on proper engineering, enables controlled drug release.

\subsection{Polymer Synthesis: Emerging Routes}

Polymer synthesis most often requires liquid environments and a variety of approaches yield defined homo-polymers, alternating, or statistical co-polymers of various monomers. MoraHuertas et al. have published an extensive review on the preparation methods of polymer-based nanocapsules for drug delivery: nanoprecipitation, emulsion-diffusion, double emulsification, emulsion-coacervation, polymer-coating, and layer-bylayer. ${ }^{[44]}$ Most of these are quite laborious and require many steps for the drug encapsulation. In recent years, polymer syntheses from vapor phase have become increasingly popular. Classically, chemical vapor deposition (CVD) routes were based on the fragmentation of specific precursor molecules by high temperatures. These are not suitable for the synthesis of polymer shells around drugs, due to the possible degradation of thermosensitive components, for example, oxidation of drug molecules. Further developments provide now more advanced polymeric vapor phase synthesis processes, which are known as initiated chemical vapor deposition (iCVD), ${ }^{[54]}$ plasma enhanced chemical vapor deposition (PE-CVD), ${ }^{[55]}$ or self-limiting techniques, such as atomic and molecular layer deposition (ALD and MLD, respectively). ${ }^{[56,57]}$ Each of these methods uses different chemical routes for the synthesis of specific polymers/matrices and, particularly, each method provides specific process-tunable properties, for instance leading to significantly lower synthesis/ reaction temperatures. One important advantage of polymer synthesis routes from the vapor-phase is the absolute lack of 
solvent: the monomers and active species are delivered pure and directly on the substrate as vapors. The elimination of the need to dissolve the polymers facilitates the encapsulation of drugs by avoiding the necessity of using multiple dissolution steps, avoids unwanted drug dissolution and mixed interfaces. In addition, no plasticizers, leachable, and surfactants are needed and this eliminates the health considerations associated with these components. The various vapor phase processes enable the production of numerous different polymer thin films with a chemistry similar to the one obtained with classic synthesis routes or leading to co-polymers with otherwise inaccessible monomer combinations, since monomers with different solubilities can be more easily copolymerized in dry processes. Copolymers of hydrophobic and hydrophilic components, highly crosslinked and fluorinated polymers are just some examples of the "unconventional" chemistries that are easily accessible by vapor methods. From these, polymers and matrices could be produced and applied in well-established processes for fabricating solid or liquid formulations, similarly to the classical solution-processed methods. However, the slower polymer processing of vapor-phase methods represents a hurdle for the replacement of conventional up-scaled routes.

The possibility to synthesize excipients/matrices from vapor phase processes enables alternative routes, distinct from standard liquid, or solid state processes, allowing the development of enhanced or even new functionalities in drug formulations. For instance, a wet (spray) coating onto pellets has an enhanced contact due to slight dissolution of the pellets at the interface with the coating, possibly reducing the mechanical stability of this solid state formulation. Contrary, in a vapor phase process, the monomers/precursor molecules mainly adsorb/ chemisorb at the dry interface reducing potential complications due to moisture increase.

Vapor processes allow also one to reproduce the geometry of the substrate because the thickness uniformity can be controlled at the nanoscale, also on grooves or microchannels, preserving the original micro/nano-structuration. This property can be used for engineering more complex devices based on nanostructured conjugates of polymers and drugs.

A summary of the advantages offered by vapor deposition methods is sketched in Figure 1. In this contribution, the current state of vapor deposited polymer/matrix investigation in pharmaceutical relevant environments and research is critically reviewed. Notwithstanding the remarkable potential of these techniques in exchanging excipients in standard processes, the focus is to highlight current trends in the development of new strategies for advanced drug encapsulation techniques aimed at the control of the drug delivery in a variety of potential applications.

\subsection{Toxicology and Approval}

Regulatory approval is a major hurdle in the introduction of any new substance within medications, cosmetics, or food and should guarantee prevention from harm to living organisms and the environment. Consequently, many tests need to be provided making the market introduction a stage of potential high commercial risk. ${ }^{[58,59]}$ For this reason, companies and developers aim at using already approved and well-established materials for the generation of new formulations, as such approval is of zero or much lower risk. For new approaches, for example, comprising vacuum deposition techniques, the improvement in functionality, and potential applications must compensate for the potential risk to justify their usage, and up to now their broad application in drug formulation is limited. Nevertheless, the large spectrum of different chemistries allows many different molecules to be combined which, in addition, can positively influences the stages of approval. For instance, using monomer units of potential low toxicity minimizes harm to living organism. Biodegradable polymers ${ }^{[60,61]}$ prevent problems of material accumulation, given that the degenerated polymer fragments are harmless. In vitro tests demonstrate that, in fact, the cytotoxic impact of most polymeric layers is very low or even absent. ${ }^{[52]}$ While such tests are limited in estimating the behavior in an extended in vivo environment where numerous biological and chemical mechanisms are additionally involved, the initial studies are very promising. This might even lead to the conclusion that, with some uncertainty, with the adoption of vapor-phase deposition processes of polymers, a very similar margin of safety is accessible when compared to conventional and more established polymeric systems.

\section{Vapor-Phase Deposition Methods}

In order to achieve controlled drug encapsulation and delivery, nanocomponents, hierarchical structures, and multilayers have to be combined in intelligent devices. In this scenario, vapor phase deposition methods, such as CVD and self-limited processes, are very appealing because they give the possibility of synthesizing polymer thin films with engineered surface properties, functionalities, and topography with high uniformity and scalability. ${ }^{[62]}$ A deep knowledge of the process allows the tuning of the properties of the deposited polymer films such as chemical composition, geometry (i.e., conformality to (nano-) structured substrates), morphology in terms of cross-linking and crystallinity degrees, durability, and thermal stability. In this review, three major vapor-phase deposition methods will be discussed in terms of synthesis mechanism and applicability to pharmaceutical environment, namely initiated CVD, plasma enhanced-CVD and atomic/molecular layer deposition. Schematics of the vapor-phase methods presented in this work are reported in Figure 2.

As afore-mentioned, polymers are conventionally synthetized in solution processes. In contrast, vapor-phase polymerizations work in conditions ranging from atmospheric pressure to mildvacuum environments, in which the monomers and active species are not dissolved in a common media, but flown as vapors and/or gases in the reaction vessel. The polymer is then formed by the adsorption of the polymer building blocks on the substrate, which is often kept at low temperature. In other words, the vapor-phase processes are completely dry. ${ }^{[63]}$

The way in which the polymer building blocks are formed from the monomer vapors is what mainly distinguishes the different vapor-phase processes. Synthetic routes like parylene polymerization require thermal energy to decompose the monomer species and initiate the growth of the polymer 


\section{Vapor-phase deposition methods}
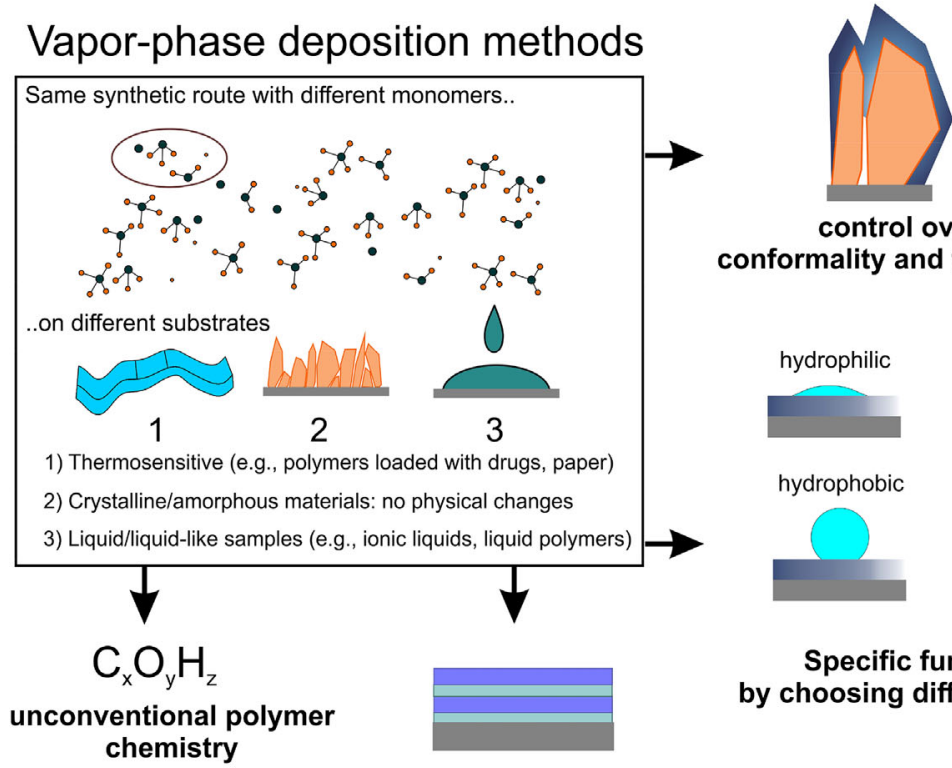

control over conformality and thickness

Multilayer structure obtained in single processes

Figure 1. Schematics of the major advantages of vapor-phase deposition methods applied for the encapsulation of drugs.

chains. ${ }^{[64]}$ Other types of CVD methods are based on the production of radicals and ions by light irradiation ${ }^{[65]}$ or by assistance of a plasma discharge. ${ }^{[55]}$ Processes like initiated chemical vapor deposition (iCVD) make use of a sacrificial molecule, called initiator, that is, thermally decomposed at relatively low temperatures, at which the monomer units are stable (Figure $2 \mathrm{~b}$ ). ${ }^{[54]}$ The so-formed radicals selectively react with the vinyl bonds of the monomer units following the mechanism of a classical radical polymerization. Therefore, only monomers bearing vinyl groups can be polymerized by iCVD a)

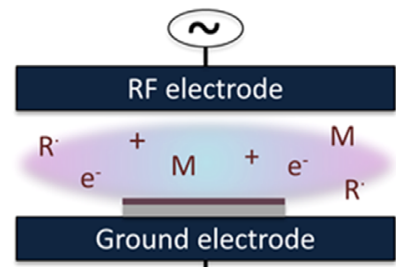

b)

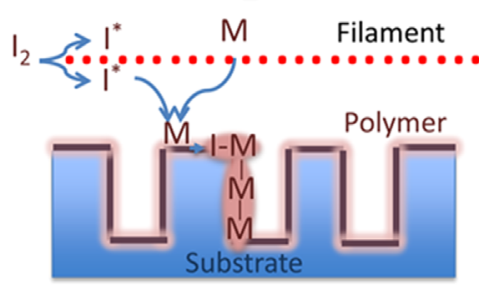<smiles>C=C(C)C(=O)OC</smiles>

methyl methacrylate<smiles>C=CCN</smiles>

allylamine c)

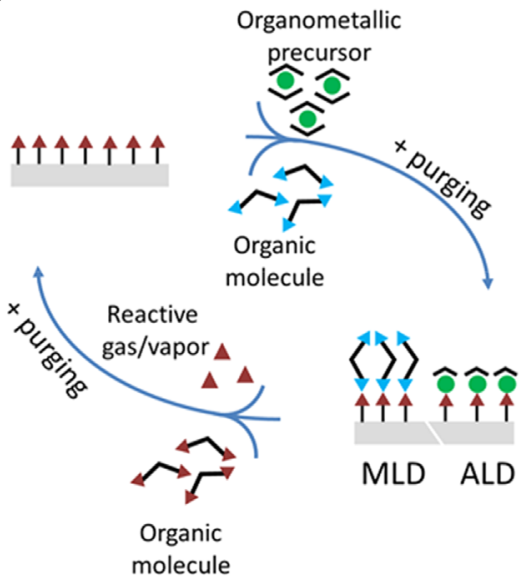<smiles>OCCO</smiles><smiles>CC[Te]CC</smiles>

ethylene glycol

diethylzinc

Figure 2. Vapor-phase deposition methods applied for the encapsulation of drugs: a) plasma enhanced chemical vapor deposition (PE-CVD), b) initiated chemical vapor deposition (iCVD), c) atomic/molecular layer deposition (ALD, MLD). At the bottom, some examples of monomers/precursors adopted with the relative chemical structure. A complete list of the molecules reported in this review is presented in Table 1. 
Table 1. Summary of the deposition methods, monomer/precursors and model drug/molecule investigated with the different strategies presented in Section 3.

\begin{tabular}{|c|c|c|c|}
\hline $\begin{array}{l}\text { Deposition } \\
\text { method }\end{array}$ & $\begin{array}{l}\text { Monomers/ } \\
\text { precursor }\end{array}$ & $\begin{array}{c}\text { Model } \\
\text { drug/molecule }\end{array}$ & Ref \\
\hline iCVD & $\begin{array}{l}\text { Methacrylic acid } \\
\text { Ethylacrylate } \\
\text { Ethylene dimethacrylate }\end{array}$ & $\begin{array}{l}\text { Fluorescein } \\
\text { Ibuprofen }\end{array}$ & \\
\hline iCVD & $\begin{array}{l}\text { Methyl methacrylate } \\
\text { Ethylene dimethacrylate }\end{array}$ & Camptothecin & \\
\hline iCVD & $\begin{array}{l}\mathrm{N} \text {-isopropylacrylamide } \\
\text { Diethylene glycol divinyl ether } \\
\text { Aminostyrene }\end{array}$ & Camptothecin & \\
\hline iCVD & $\begin{array}{l}\text { 2-hydroxyethyl methacrylate } \\
\text { Methacrylic acid } \\
\text { Perfluorodecyl acrylate }\end{array}$ & Clotrimazole & \\
\hline PE-CVD & Allyl alcohol & Acetylsalicylic acid & \\
\hline PE-CVD & Tetramethylcyclo-tetrasiloxane & $\begin{array}{l}\text { Daunomycin } \\
\text { Rapamycin } \\
\text { NPC-15199 }\end{array}$ & \\
\hline PE-CVD & $\mathrm{N}$-heptylamine & Levofloxacin & {$[93$} \\
\hline PE-CVD & $\begin{array}{l}\text { Acrylic acid } \\
\text { Hexamethyldisiloxane }\end{array}$ & Methylene Blue & \\
\hline PE-CVD & $\begin{array}{l}\text { Polydimethylsiloxane } \\
\text { Tetraethyl orthosilicate }\end{array}$ & Rifampicin & \\
\hline PE-CVD & $\begin{array}{l}\varepsilon \text {-caprolactone } \\
\text { 2-methoxyethyl ether }\end{array}$ & $\begin{array}{l}\text { Methylene Blue } \\
\text { Cisplatin }\end{array}$ & $\begin{array}{c}{[99} \\
{[100}\end{array}$ \\
\hline PE-CVD & $\begin{array}{l}\text { Air-fed plasma } \\
\text { Diethylene glycol dimethyl } \\
\text { ether }\end{array}$ & Ampicillin & \\
\hline PE-CVD & Tetraglyme & Ampicillin & {$[1$} \\
\hline PE-CVD & Allylamine & Vancomycin & \\
\hline PE-CVD & Perfluorooctane & Camptothecin & \\
\hline PE-CVD & $\begin{array}{l}\varepsilon \text {-caprolactone } \\
\text { 2-methoxyethyl ether }\end{array}$ & Simvastatin acid & \\
\hline PE-CVD & Perfluorohexane & Ketoprofen & \\
\hline PE-CVD & Ethylene & Lysozyme & \\
\hline PE-CVD & Ethylene & $\begin{array}{l}\text { Vancomycin } \\
\text { Fluorescein }\end{array}$ & \\
\hline PE-CVD & Acrylic acid & Doxorubicin & \\
\hline PE-CVD & Titanium (IV) tetraisopropoxide & $\begin{array}{l}\text { a-lipoic acid } \\
\text { Heparin } \\
\text { Abciximab }\end{array}$ & \\
\hline PE-CVD & Helium & Doxycycline hyclate & {$[1$} \\
\hline PE-CVD & $\operatorname{Ar}$ & $\begin{array}{l}\text { Salicylamide } \\
\text { Theophylline } \\
\text { Diclofenac sodium }\end{array}$ & $\begin{array}{l}{[12} \\
{[122} \\
{[12}\end{array}$ \\
\hline PE-CVD & $\begin{array}{l}\mathrm{Ar} \\
\mathrm{O}_{2} \\
\mathrm{~N}_{2}\end{array}$ & Curcumin & \\
\hline ALD & $\begin{array}{l}\text { Trimethyl aluminum } \\
\text { Deionized water }\end{array}$ & Ketoprofen & \\
\hline ALD & Trimethylaluminum & Acetaminophen & \\
\hline
\end{tabular}

(Continued)
Table 1. (Continued)

\begin{tabular}{llll}
\hline $\begin{array}{l}\text { Deposition } \\
\text { method }\end{array}$ & \multicolumn{1}{c}{$\begin{array}{c}\text { Monomers/ } \\
\text { precursor }\end{array}$} & \multicolumn{1}{c}{$\begin{array}{c}\text { Model } \\
\text { drug/molecule }\end{array}$} & Ref. \\
\hline & Titanium tetrachloride & & \\
& $\begin{array}{l}\text { Titanium(IV)isopropoxide } \\
\text { Diethylzinc }\end{array}$ & & \\
& Deionized water. & & \\
& Diethylzinc & Fluorescein & [108]
\end{tabular}

and the polymers obtained have a great chemical specificity and a predictable chemical structure. During the iCVD process, copolymerization occurs due to simultaneous feeding of the different co-monomers into the reactor. The ratio of the monomer partial pressures and the saturation pressure at the substrate temperature (i.e., $\left.P_{M} / P_{\text {sat }}\right)^{[54]}$ is used as a quantification of the relative mass amount of each component in the reaction chamber. By varying the $P_{M} / P_{\text {sat }}$ ratio, polymers with adjustable co-monomer ratios can be obtained. This approach allows the control of the chemical composition and, thus properties of the deposited polymer, such as cross-linking and solubility, amongst many others. The total retention of the monomer functionalities can be a highly desired property for drug encapsulation and delivery. The polymer functional groups can be used for immobilization of the drug or for targeted drug delivery (e.g., enteric release by $\mathrm{pH}$-responsive polymer encapsulants ${ }^{[66]}$ ).

On the other hand, in a polymerization enhanced by plasma there is no limitation on which monomers can be adopted. In a plasma discharge, the main sources of fragmentation of the monomer vapors are electronic collisions, which are nonselective toward certain types of chemical bonds (Figure 2a). All the bonds of the gaseous species feeding the plasma can be fragmented and even polymers of methane can be obtained. ${ }^{[67]}$ As a consequence, the number of reaction pathways that monomer species can undergo during a plasma polymerization is rather large and the chemical composition of the final polymers is difficult to predict. ${ }^{[68]}$ In addition to the fragmentation in the gas phase, the growing polymer is known to be subject to positive ion bombardment. This creates rearrangements of bonds, cleavages of labile groups, and formation of crosslinks and branches, increasing the number of possible reaction pathways. To limit the fragmentation of the monomer structures and the positive ion bombardment, "soft" conditions can be used, for example, high pressure, low, and often pulsed plasma power, resulting, as a drawback, in low deposition rates. ${ }^{[69]}$ Another strategy to reduce plasma damage to the growing material is to use remote plasmas, in which the polymer is grown on a substrate not directly exposed to the plasma area. One of the major drawbacks in using plasma-based techniques is the possible damage/fragmentation of the drug molecules, when these are directly exposed to the plasma, which in turn leads to its deactivation or toxicity. The afore-mentioned soft process conditions, therefore, have to be adopted in order to keep intact the drug molecule structure.

Very recently, self-limiting deposition methods have become an increasingly adopted solution for the encapsulation of drugs. Polymers or inorganic matrices with very predictable chemical 
structures can be obtained also by molecular layer depositions $(\mathrm{MLD})^{[57]}$ and atomic layer depositions (ALD), ${ }^{[70]}$ respectively. MLD and ALD methods are based on sequential self-limiting surface reactions, yielding thickness control at the molecular level or atomic level for ALD (Figure 2c). The thin films are obtained by exposing the substrate surface to different cycles of two or more precursor doses alternated with purging gas doses to remove unreacted precursor molecules from the gas phase and leave only those that are chemisorbed on the surface. In MLD, the two precursors are generally organic molecules that react with each other through coupling reactions. The selflimiting nature of these reactions leads to a film formed by one molecular layer at the time. In ALD, one precursor is metalorganic and the other is generally an oxidant (e.g., water vapor, ozone, or oxygen plasma). The latter serves as oxidant for the organic component of the precursor and yield a pure oxide film. The self-limiting surface reactions of the MLD and ALD processes result in very high conformality and sub-nanometer thickness control, but extremely low deposition rates ${ }^{\mathrm{a}}$. ${ }^{\text {[1] }}$

Conformality is the efficiency of a thin film to coat tridimensional features of the substrate with a uniform thickness. In the next sections, it will be demonstrated how this property can be crucial for drug encapsulation and device fabrication for drug delivery. Generally, high drug loading can be achieved in cavities or channels of membranes ${ }^{[72]}$ with a specific pore size or on nanopillars ${ }^{[73]}$ or nanoparticles. ${ }^{[74]}$ In these cases, a thin polymer film that preserves the topography of the substrate at the micro and nano-scale can be highly beneficial. Together with MLD/ALD, also iCVD has shown high efficiency in evenly covering complex geometrical features. ${ }^{[63,75]}$ On the contrary, polymers obtained from solution synthesis often show meniscus formation or de-wetting, liquid thinning, and other solvent surface tension effects, which alter the profile of the coating on the substrate. Plasma polymers have also lower level of conformality than polymers deposited by iCVD and MLD/ALD, due to some shadowing and border effects that can alter the growth of the polymer on tridimensional substrates.

All the above-mentioned vapor-phase processes operate at low temperatures, with the consequence that also delicate substrates such as plastic or paper can be easily coated. Particularly in the field of drug encapsulation, this is a great advantage, since it allows also the coating of thin molecular layers of drugs, without evaporation due to high processing temperatures and without inclusion of solvents, with the only requirement being that the drug needs to sustain the mild vacuum. ${ }^{[76]}$ Multilayered structures can also be easily obtained by vapor-phase methods (see Figure 1), since the vapor deposition does not require to seek and use orthogonal solvents for the different polymers ${ }^{\mathrm{b}}$.

\footnotetext{
${ }^{a}$ It is worth mentioning that a faster self-limiting method that overcomes the very low deposition rate of classical ALD is available, i.e., spatial ALD. In this configuration, the precursors are spatially and not temporally separated, increasing the overall deposition rate. Further details can be found in the literature. ${ }^{148]}$

${ }^{b}$ Here orthogonal refers to the fact that a solvent might be a good solvent for one component while an antisolvent for the other.
}

\section{Strategies for Drug Encapsulation and Controlled Delivery}

The recent applications of vapor-phase methods in controlling drug release are following in principle three major strategies, which are: encapsulation, embedding, and surface modification/ activation. Schemes of these approaches are given in Figure 3.

\subsection{Encapsulation}

One basic and most common strategy in controlled drug release is to encapsulate drugs with layers made from organic (generally polymers), or inorganic materials. As afore-mentioned, such an encapsulating shell often acts then as diffusion membrane in order to have a timed release and/or as protective barrier, which allows the drug release to occur when specific conditions are met $^{\text {[77] }}$ (e.g., barrier disintegration at a specific site in the body). Several vapor phase techniques have been employed for the direct encapsulation of drugs or drug loaded media, taking into account the possibility to treat a vast variety of substrates, such as solid surfaces, nanostructured/engineered surfaces, particles, but also on delicate substrates like amorphous drug films ${ }^{[64,66,76,78]}$ or liquid thin films. ${ }^{[79]}$

\subsubsection{Encapsulant-Controlled Drug Release}

Initiated CVD has been the method of choice when the retention of functional groups was sought, that is, when specific functionalities of the polymer such as stimuli responsiveness are desired. ${ }^{[80-82]}$ Lau et al. ${ }^{[66]}$ investigated the encapsulation of ibuprofen microcrystals with smart polymers derived from an iCVD process. Methacrylic acid (MA) was co-polymerized with different cross-linking agents, namely ethylacrylate (EA) and ethylene dimethacrylate (EDMA) which then resulted in a $\mathrm{pH}$ responsive polymeric layer system. In order to verify the $\mathrm{pH}$ responsiveness of this iCVD co-polymer, the poly(MAA-EDMA) was immersed in various $\mathrm{pH}$ buffers, and its swelling behavior was determined by measuring the variation in thickness using spectroscopic ellipsometry (SE, Figure 4a). SE can be used to derive the relative change in co-polymer film thickness $(\Delta d)$ between initial dry films $\left(d_{0}\right)$ and the final swollen films upon immersion in various buffers of distinct $\mathrm{pH}$. At low $\mathrm{pH}$, the copolymer showed a limited swelling, keeping a denser matrix (swelling $\approx 5 \%$ ). When these layers are exposed to higher $\mathrm{pH}$ solutions ( $\mathrm{pH}$ 6.5-7), an abrupt variation in thickness was observed and the co-polymer showed significant swelling of more than 30\% (Figure 4a). Furthermore, such swelling is reversible which even allows multiple swelling and deswelling cycles to be employed.

Differences in swelling behavior can often be used to selectively release an encapsulated drug when determined conditions occur, like specific $\mathrm{pH}$ and temperatures, thus enabling targeting specific sites or organs in mammals. ${ }^{[77]}$

In order to verify the effect of swelling on drug release, the model drug ibuprofen was encapsulated with poly(MAAEDMA). Both model systems showed a different release kinetic when going from acidic to neutral $\mathrm{pH}$. As clarified in 
a)

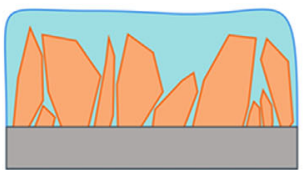

b)

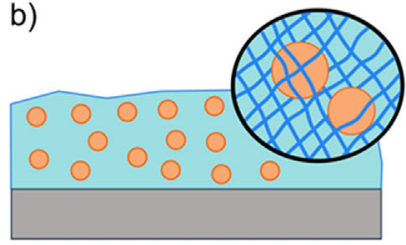

c)

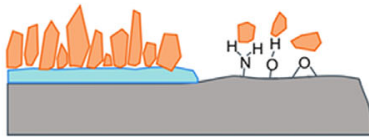

Figure 3. Schematics of the strategies for the controlled drug delivery: a) encapsulation, b) embedding, c) surface modification/activation. The polymer is represented in light blue, while the drug in orange.

Figure $4 \mathrm{~b}$ and $\mathrm{c}$, the co-polymer, acting as a diffusion barrier, significantly hinders the model drug/molecule release at low $\mathrm{pH}$, while allowing a faster release when the $\mathrm{pH}$ is increased close to neutral values. It is worth noticing that, with the drug molecule adopted, differences in its solubility were already visible at different $\mathrm{pH}$ when no encapsulation was present.

As mentioned in the previous section, the possibility to conformally coat complex tridimensional geometries allows the engineering of more advanced drug delivery systems. iCVD has been demonstrated to achieve highly controlled and conformal layers, coating complex 3D geometries. ${ }^{[83-86]}$ McInnes et al. ${ }^{[73]}$ adopted iCVD deposited $\mathrm{pH}$ responsive polymers for the encapsulation of nanoporous drug delivery vehicles, that is, drug loaded porous silicon (pSi). Camptothecin (CPT) was chosen as the model drug. ${ }^{[87]}$ After filling the pores of $\mathrm{pSi}$ with the drug, methyl methacrylate (MAA) was then polymerized on top of such loaded substrates. EDMA was adopted as crosslinking agent. A schematic of the device is presented in Figure 5a. As for the encapsulation of ibuprofen, at low $\mathrm{pH}$ the cross-linked film will act as diffusion barrier, while at neutral $\mathrm{pH}$ the film will allow the diffusion of the drug out of the porous hosts. Figure 5b shows the release profile of CPT at $\mathrm{pH} 1.8$ and 7.4. In contrast to the ibuprofen microcrystals shown by Lau et al., the drug release of CPT here from uncoated loaded-pSi was independent from the $\mathrm{pH}$ value of the solution, and about $80 \%$ dissolved within $17 \mathrm{~h}$. It is important to note that CPT is shown to be a better model substance for getting insights into the diffusion properties of a specific coating, since any deviating property of the buffer solution on the formulation and its drug release can be solely attributed to the coating. This means that the samples coated with poly(MAA-co-EDMA) reveals two significantly different drug release profiles which can now only be relatable to the polymer swelling in the different environments; in $17 \mathrm{~h}, 10.7 \%$ of the drug was released at $\mathrm{pH} 1.8$, while higher values, $44.5 \%$, of the drug was released at $\mathrm{pH}=7.4$.

The authors also compared the kinetics of drug release for coated or uncoated pSi. Often, two different regions can be distinguished in a drug release profile, when the drug-loaded layer is put in contact with a solvent. A first sudden release of drug is often reported, followed by a zero order kinetics. The sudden release is then usually reported as burst release. ${ }^{[88]}$ Burst release leads to a very high initial drug release, and, in turn, local high concentration, causing often localized irritation, or even systemic problems and should for most drug formulation be prevented. Moreover, it reduces the lifetime of the drug release device, at the same time decreasing the amount of drug that can effectively be controlled. Again, an iCVD layer can help reducing burst release, as visible in Figure 5b. When poly(MAA-co-EDMA) is deposited, the burst release was strongly reduced. This effect is enhanced at lower $\mathrm{pH}$. As a consequence, a slow release might be achieved in the gastric juice while intestinal fluid might reduce this less effectively. This reduction was used as a proof for the possibility of tuning the drug release kinetics as a function of $\mathrm{pH}$ conditions

In a separate study, McInnes et al. ${ }^{[74]}$ investigated also the fabrication and characterization of a pSi drug delivery vehicle capped with an alternative polymer, which was temperatureresponsive. Thermo-responsive polymers are a class of smart polymers characterized by a volume phase transition at a certain temperature, which causes a sudden change in the polymer solvation state. Polymers which become insoluble upon heating have a lower critical solution temperature (LCST). On the contrary, polymers that become soluble upon heating, have a so-called upper critical solution temperature (UCST). ${ }^{[81,89]}$ Poly N-isopropylacrylamide (polyNIPAAm) is one of the most studied thermos-responsive polymers and it is characterized by a LCST of $32^{\circ} \mathrm{C}$. In their investigation, McInnes et al. adopted poly(N- isopropylacrylamide-co-diethylene glycol divinyl ether) (pNIPAAm-co-DEGDVE) and compared the performance of this with a non-stimulusresponsive poly-aminostyrene (pAS). Both polymers were deposited by means of iCVD. In a similar fashion as for the pH-responsive polymer capped CPT-loaded pSi, the CPT release was observed for the uncoated and coated pSi at two different temperatures, 37 and $25^{\circ} \mathrm{C}$, higher and lower values than the polymer LCST, respectively. After $16 \mathrm{~h}$, the amount of CPT released from the pSi coated with pNIPAM-co-DEGDVE was $52.8 \%$ at $37^{\circ} \mathrm{C}$ and $36.1 \%$ at $25^{\circ} \mathrm{C}$. This points out that more $\mathrm{CPT}$ is released as the polymer collapses. The authors speculate that, considering the conformal nature of the coatings, the opening of the pSi pores widens as the polymer contracts, enabling the drug to diffuse out at a higher rate. This is also confirmed by the burst release present at the initial stages of the diffusion, which is higher at $37^{\circ} \mathrm{C}$ compared to $25^{\circ} \mathrm{C}$. However, the authors give an alternative explanation, that is, the ejection of CPT molecules close to the pore openings or on top of the porous layer. The non-responsive polymer polyAS showed no difference in release kinetics on temperature variation, confirming that the differences observed with pNIPAMco-DEGDVE are attributable to the polymer thermo-responsive properties.

Next to iCVD, plasma enhanced CVD has also been employed for the synthesis of protective coatings to target controlled drug release. Compared to iCVD layers, PE-CVD deposited polymers generally show a higher degree of cross-linking, due to its underlying growing mechanism involving radicals and other 
a)

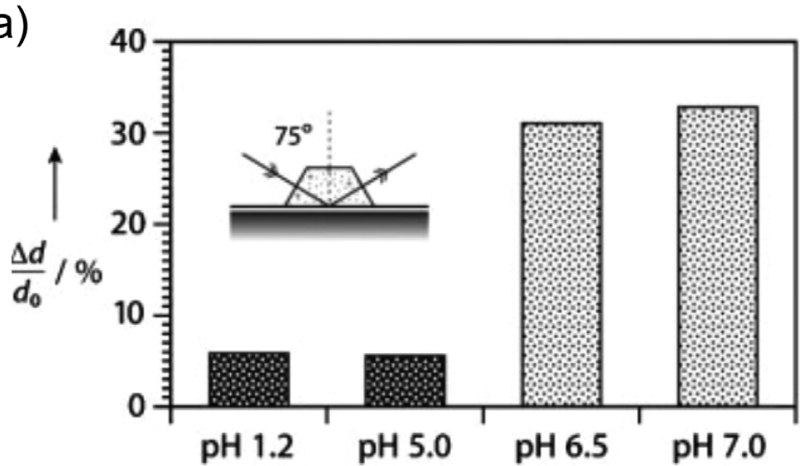

b)

c)
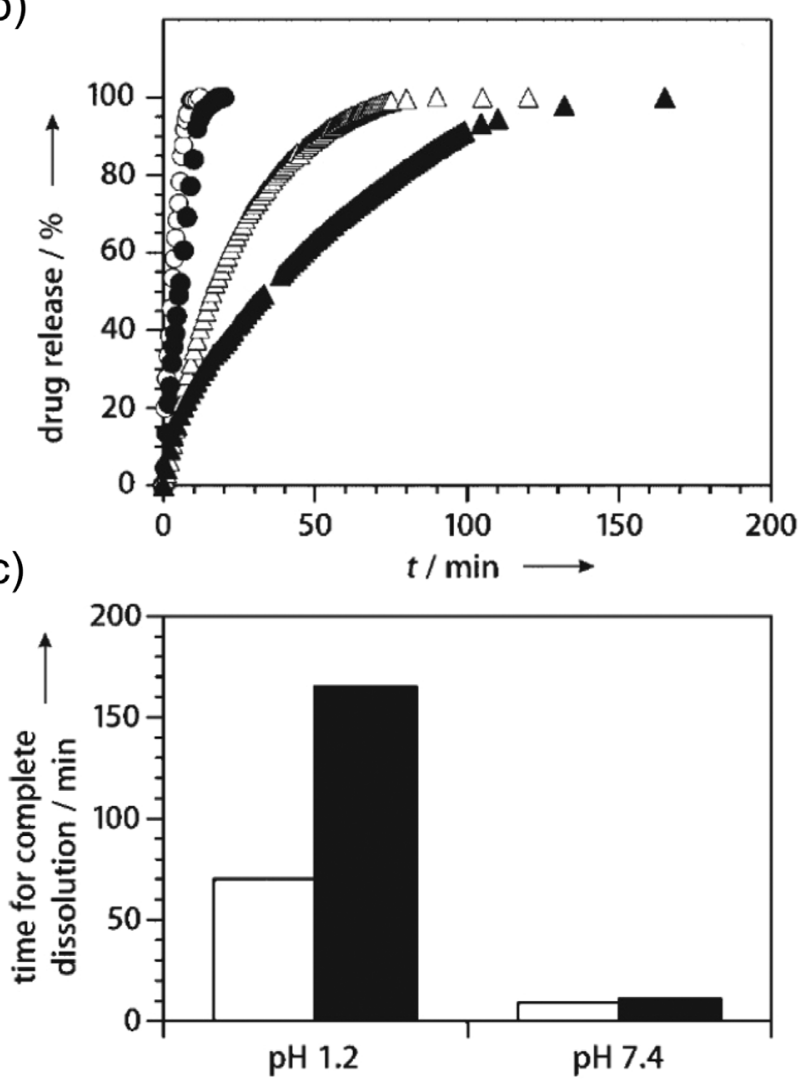

Figure 4. a) Spectroscopic ellipsometry analysis of iCVD p(MAA-EDMA). Change in the relative polymer film thickness $(\Delta d)$ upon exposure to different $\mathrm{pH}$ buffer soaks, normalized to the dry thickness $\left(d_{0}\right)$. b) Controlled ibuprofen release without coating (open symbols) and with the iCVD p(MAA-EDMA) coating (filled symbols), at $\mathrm{pH} 1.2$ (triangle) and $\mathrm{pH}$ 7.4 (circle); c) Time for complete release in different $\mathrm{pH}$ buffers for coated (unfilled bar) and uncoated (filled bar) ibuprofen samples. Reproduced with permission from ref.[54] Copyright (C) 2007 WILEY-VCH Verlag $\mathrm{GmbH} \&$ Co. KGaA, Weinheim.

(fragmented) reactive species. ${ }^{[55]}$ Furthermore, they show a better adhesion to the treated substrate, due to the modification of the substrate surface in the initial stages of the plasma polymerization. However, due to the directionality of the plasma ions (actively participating in the film growth) and the high reactivity of the (fragmented) radicals, lower conformality can be achieved, limiting their use on high aspect-ratio nanostructured substrates. Finally, the highly reactive plasma environment can modify the surface of the treated samples, changing their chemical nature. It should be noted, that direct treatments of (bio-)active molecules can, therefore, lead to partial inactivity or toxicity, ${ }^{[90]}$ which might reduce its applicability.

The first work on direct drug encapsulation by means of PECVD was reported in 2005 by Susut and Timmons. ${ }^{[1]}$ In their work, acetylsalicylic acid (aspirin) microcrystal were coated in a PE-CVD reactor with plasma polymerized allyl alcohol. In order to retain the precursor chemical structure and limit the possible damage of the plasma, a 'soft' pulsed PE-CVD approach was adopted. In this approach, during the deposition, the plasma is pulsed at regular intervals (duty cycle, DC), allowing the activation of the depositing species and at the same time limiting its fragmentation. Moreover, the plasma exposure time on the treated samples is limited, reducing plasma induced surface modifications. An RF duty cycle is generally reported as in Equation 1.

$\mathrm{DC}=\frac{\tau_{\mathrm{on}}}{\tau_{\mathrm{on}}+\tau_{\mathrm{off}}}$

where $\tau_{\text {on }}$ and $\tau_{\text {off }}$ are the plasma on and off times, respectively. The polymer properties were investigated as a function of three processing variables, namely RF power, coating time and RF duty cycle. This demonstrates the versatility of vapor-deposited polymers in general, as many processing parameters can be adjusted to meet the application-specific requirements. The matrix properties can be easily varied and adapted to the system to encapsulate. Acetyl-salicylic acid release rates were measured with UV-Vis spectroscopy. The release rates were found to decrease as the power input, the coating time and plasma duty cycles are increased. Increasing the coating time leads to higher thicknesses that slow the out-diffusion of the drug molecules. The power input and duty cycle act instead on the cross-linking, which reduces the overall free volume of the polymer, in turn hindering the drug release. Additionally, in their work thin-layer chromatography (TLC) was adopted to determine if undesirable conversion of some drug molecules to other compounds occurred due to plasma exposure. The TLC results suggest that negligible amounts of drug molecules are converted to other compounds during the coating process, showing the feasibility of pulsed PE-CVD in the direct coating of drug molecules.

Similar conclusions are reported by Zamora et al.. ${ }^{[80,92]}$ where three different drugs are coated with a plasma organosilicon polymer. In their work, a direct plasma without any pulse was adopted. However, the authors do not comment on the stability of the drugs in the conditions explored.

\subsubsection{Encapsulant-Controlled Interaction with External Environment}

Together with a better control on the release of active molecules, encapsulating polymers can be used for changing the surface interaction with external environments, for example, antifouling chemistry or improved wettability. Vasilev et al. ${ }^{[33,94]}$ reported on the plasma polymerization of $n$-heptylamine for the 
a)

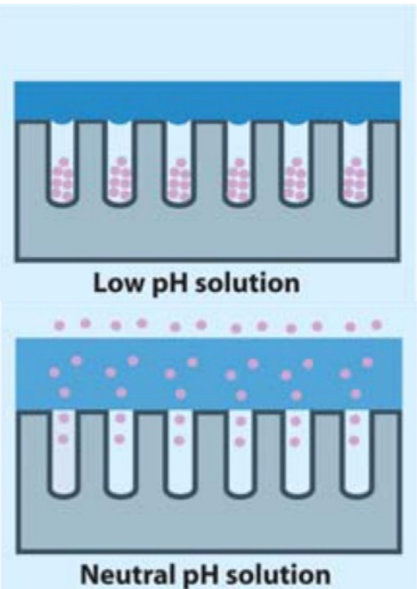

b)

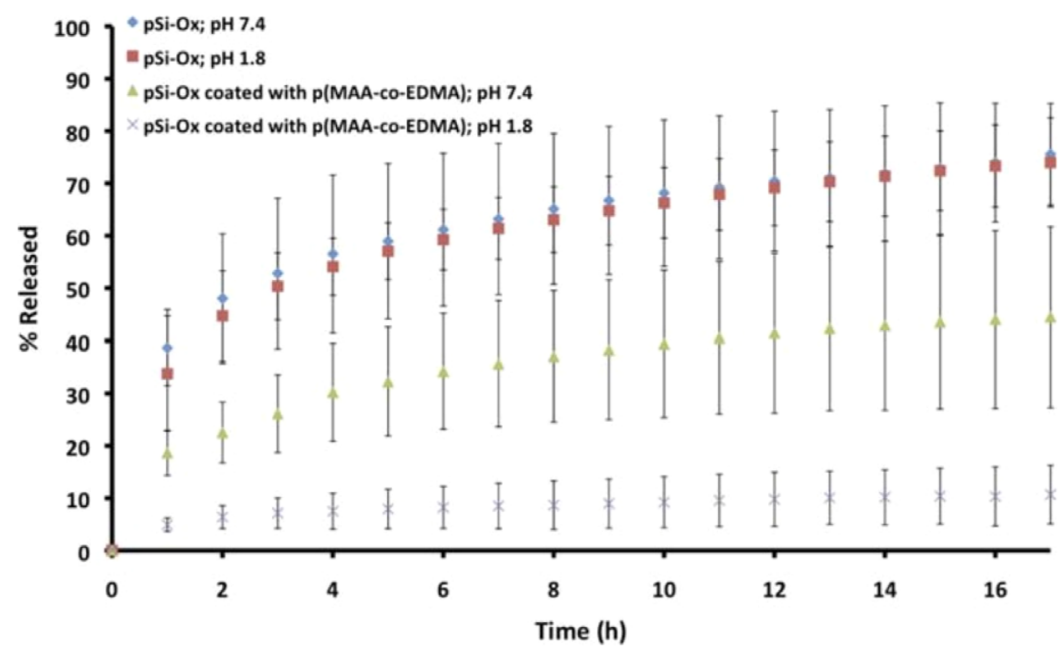

Figure 5. a) Schematics of the pSi coated with the iCVD polymer and the mechanism of release at different $\mathrm{pH}$. b) Drug release curves for CPT from uncoated $\mathrm{pSi}$ and after coating with $\mathrm{p}$ (MAA-co-EDMA) at a $\mathrm{pH}$ of 1.8 and 7.4. The $\mathrm{p}$ (MAA-co-EDMA) coating enabled the controlled pH-dependent release of the drug from the drug delivery system. Adapted with permission from ref.[61] Copyright (2012) American Chemical Society.

encapsulation of drop-casted levofloxacin. In their study, a first ultra-thin $(10 \mathrm{~nm})$ plasma polymer layer is applied on the surface of untreated samples (quartz substrates). The role of this layer is to provide defined surface wettability before the application of the drug solution, as on some materials insufficient wetting might cause inhomogeneous spreading. As they report, for the medical implant materials titanium and polyethylene, drying of the applied drop of drug solution would likely result in different shapes and sizes of the drug particles owing to different capillary forces acting on surfaces with different surface energies. The drop-casted levofloxacin is then encapsulated with a thicker $n$ heptylamine plasma polymer. The authors reported on faster first order kinetics as a function of the loaded amount of the drug, which in the case studied results in bigger levofloxacin crystals. In general, smaller particles provide faster dissolution on account of larger surface area but the reason for a faster release here has to be attributed to the poor conformality of the plasma polymer at the crystals surface. Especially, as the crystal surfaces increased their roughness/nano-structuration, the coating grows even less conformal and thinner in some crystal facets, thus allowing for a faster release. ${ }^{[93]}$

In a similar fashion, Zanini et al. ${ }^{[95]}$ reported on the loading of a model dye, methylene blue (MB), on polypropylene (PP) substrate pre-treated with plasma-induced graft polymerization of acrylic acid (AAc). Due to the presence of carboxylic groups, PP-AAc films immersed in a buffer solution were found to adsorb one order of magnitude more cationic MB compared to the untreated PP. The PP-AAc loaded samples were then coated with a plasma polymer obtained with hexamethyldisiloxane (HMDSO)-fed plasma. Together with the intrinsic diffusion barrier properties of the organosilicon polymer, its hydrophobicity further enhanced the control over the dye release. Water diffusion into the layer was hindered, delaying the MB outdiffusion. Additionally, in order to get further insights into the kinetics of diffusion, the MB release over time was modelled with the equation proposed by Ritger and Peppas, as reported in Equation 2. ${ }^{[96,97]}$

$M_{t} / M_{\text {tot }}=k t^{n}$

where $M_{t}$ is the mass of compound released at any given time $t$, $M_{\text {tot }}$ is the total mass of compound loaded, $t$ is the release time, $k$ is the kinetic constant of the system, and $n$ is a characteristic exponent for the release. This equation is commonly used to describe the release kinetics of a compound and agrees only for the first $60 \%$ of the mass dissoluted. When $n$ is 0.5 , the compound is considered diffusing with Fickian behavior. For $n$ of 0.25 , the behavior is called Case II diffusion. Finally, anomalous transport behavior is known as non-Fickian diffusion and it is intermediate between Fickian and Case II.

For HMDSO-polymer coated PP-AAc, the value of $n$ was found in the non-Fickian diffusion case (0.73). The authors explain this behavior accounting for the granular structure of the HMDSO coatings, in which intergranular pores are relatively large compared to the model molecule.

A similar sandwich-like structure has been recently investigated by Dowling et al. ${ }^{[98]}$ In this case, atmospheric pressure plasma CVD was adopted. Polydimethylsiloxane (PDMS) and tetraethyl orthosilicate (TEOS) were used as organosilicon precursors. The wettability of the polystyrene substrate toward the drug molecule under study (Rifampicin) was enhanced by the deposition of a TEOS-polymer and then capped with PDMSpolymer. The possibility to achieve hydrophilic surfaces by means of plasma polymers modification could increase the number of accessible substrates that can potentially be loaded with drug molecules.

The possibility with vapor-phase methods to easily engineer multilayer structures is well represented by the works of Bhatt et al. ${ }^{[99,100]}$ In their investigations, in vitro, ${ }^{[99]}$ and in vivo ${ }^{[100]}$ studies were performed on pulsed inductive PE-CVD polymers 
of poly ( $\varepsilon$-caprolactone)-poly (ethylene glycole) (PCL-co-PEG) obtained with $\varepsilon$-caprolactone $(\varepsilon$-CL) in the presence of 2 methoxyethyl ether (DEGME). The multilayer structure consisted of a buffer layer of poly(DEGME) to increase the surface hydrophilicity and improve the uniformity of the drug layer. The drug (cisplatin) or the model molecule (MB) were loaded on the first poly(DEGME) layer. The encapsulation layer comprised three layers. A first PCL-co-PEG layer in direct contact with the loaded molecules was deposited adopting a soft pulsed discharge to minimize the possible damage on the loaded molecule occurring upon plasma exposure. A second PCL-Co-PEG was deposited in continuous plasma mode, in order to obtain a denser and more cross-linked layer to work as release control barrier. Finally, a third PCL-co-PEG coating deposited at mild plasma conditions to obtain a functionalized layer. This layer was meant to control the biological activities such as the cell adhesion and proliferation, and cell migration, on the surface owing to the retention of ether groups available on the surface of the co-polymer coatings. This multi-layered system allowed the study of the drug release decoupled from the cell proliferation, due to the separate roles of the second and third layer. The kinetics of the drug release was found to be a function of the layer thickness ${ }^{[99]}$ shifting from a pure zero order diffusion to a non-Fickian regime concluding from the Peppas equation (Eq. 2). ${ }^{[96,97]}$

Furthermore, the in vitro study showed that cisplatin incorporated with the multilayer coatings had higher cytotoxicity when compared to cisplatin alone. These results point out that the third PEG-rich PCL-PEG co-polymer coating offers a surface with tailorable cell surface interactions without affecting the barrier layer dependent drug release kinetics.

For the in vivo studies, histological examinations were performed on liver tissues, which were in contact with cisplatin loaded plasma polymerized cellophane implants. Hepatocellular vacuolization and cisplatin induced inflammation of cells were shown. As compared to untreated cellophane implanted on liver tissues, the apoptotic cells were found to remain in the liver tissues which were in contact with cisplatin loaded implants. ${ }^{[100]}$

\subsubsection{Encapsulation on Tridimensional Structures}

As already discussed for iCVD deposited encapsulating polymers, vapor-phase methods can attain high level of conformality, allowing the treatment of complex 3D structures. Despite the poorer conformality of PE-CVD systems when compared to other methods such as iCVD and ALD (later discussed), PE-CVD methods have been successfully used for the treatment of more complex tridimensional structures. Labay et al. ${ }^{[101]}$ reported on the treatment of PP meshes adopting atmospheric PE-CVD treatment. Pre-treatment of the meshes with air-fed plasma was adopted to increase the hydrophilicity of the PP surface and, in turn, the loading of drug (ampicillin). A PEG-like polymer is then adopted to maintain unchanged fibroblast properties such as chemotaxis or adhesion with respect to untreated PP meshes. Vasilev et al. ${ }^{[102]}$ coated vancomycin loaded anodic alumina oxide (AAO) layers using PE-CVD allylamine polymers. By accurately changing the plasma polymerization time, the AAO pore width could be reduced in a controlled way. The drug release was found to scale with the reduced pore diameter, and the amount of drug subject to initial burst release decreases with the reduction in the pore opening. Afterwards, zero order release was found and attributed to drug positioned deeper in the pores. Also the example discussed in section 3.1.1 (Figure 5), falls in the category of encapsulation on tridimensional structures ( $\mathrm{pSi}$ ). Furthermore, also porous silicon microparticles (pSi-MP) were treated with a perfluorooctane (PFO)-fed plasma polymer by McInnes et al. ${ }^{[103]}$ The successful loading of the drug (camptothecin) and subsequent coating of the loaded pSi-MP is demonstrated. The coating is shown to markedly slow down drug release, which can be further adjusted by altering the plasma deposition time. 2 -fold to 100 -fold slower drug release is demonstrated, confirmed by in vitro experiments. Human neuroblastoma showed delayed cell death for all coated particles.

Other more complex 3D structures have been treated with PECVD methods. Calcium phosphate (CaP) scaffolds loaded with drugs were used as substrates for PE-CVD polymers by Canal et $\mathrm{al}^{\left[{ }^{[104]}\right.}$ and Labay et ${ }^{[105]}$ Canal et al. reported on the treatment of two different scaffolds, Calcium Deficient Hydroxyapatite (CDHA) or high-temperature sintered $\beta$-Tricalcium Phosphate $(\beta$-TCP), characterized by different available surface areas and porosity (Figure 6). ${ }^{[104]}$ Simvastatin acid (SVA) was incorporated to the CaP scaffolds. The authors deposited biocompatible PCL-co-PEG by means of PE-CVD on both CaPs and investigated the polymer thickness and coated depth as a function of the different available surface areas. The study shows that this coating significantly changed the surface topography of the CaP materials, leading to the formation of bush or worm-like structures (Figure 6). Furthermore, when the coating was able to grow deep into the scaffold ( $\beta$-TCP), the release of the loaded drug was completely hindered. Contrary, when the coating grew only on top of the 3D structure (as in the case of the CDHA scaffold), the drug release occurs and is found scalable with the coating thickness, in agreement with the previous findings using the same plasma treatment. ${ }^{[99]}$

Labay et al. ${ }^{[105]}$ deposited PEG-like coatings on $\beta$-TCP using diglyme as precursor in the plasma process. The scaffolds were previously loaded with ampicillin and release experiments were carried out as a function of the plasma process parameters. It is worth noticing that the biological activity of ampicillin was also reported after plasma polymerization. The antibiotic showed a retention of its activity, confirming the non-significant impact the plasma treatment has on loaded drugs, also when supported on complex 3D structures.

Next to polymer encapsulation, very recently vapor-phase deposited metal-oxides have been adopted as drug release barriers, either directly deposited on the drug crystals ${ }^{[106]}$ or on drug loaded polymers. ${ }^{[107,108]}$ Atomic layer deposition (ALD) has been the method of choice for the deposition of ultra-thin conformal metal oxides, showing promising results in terms of controlled release which might differ from those obtainable from organic derived layers. As afore-mentioned, ALD is an effective method for the coating/surface modification of high surface area and highly porous materials. Due to the diffusive and self-limiting nature of its growth mechanism, this technique allows for sub-atomic control of layer thickness, in this being superior to the other vapor-phase methods. Vogel et al. ${ }^{[107]}$ reported the treatment of poly(vinyl alcohol) (PVA) 


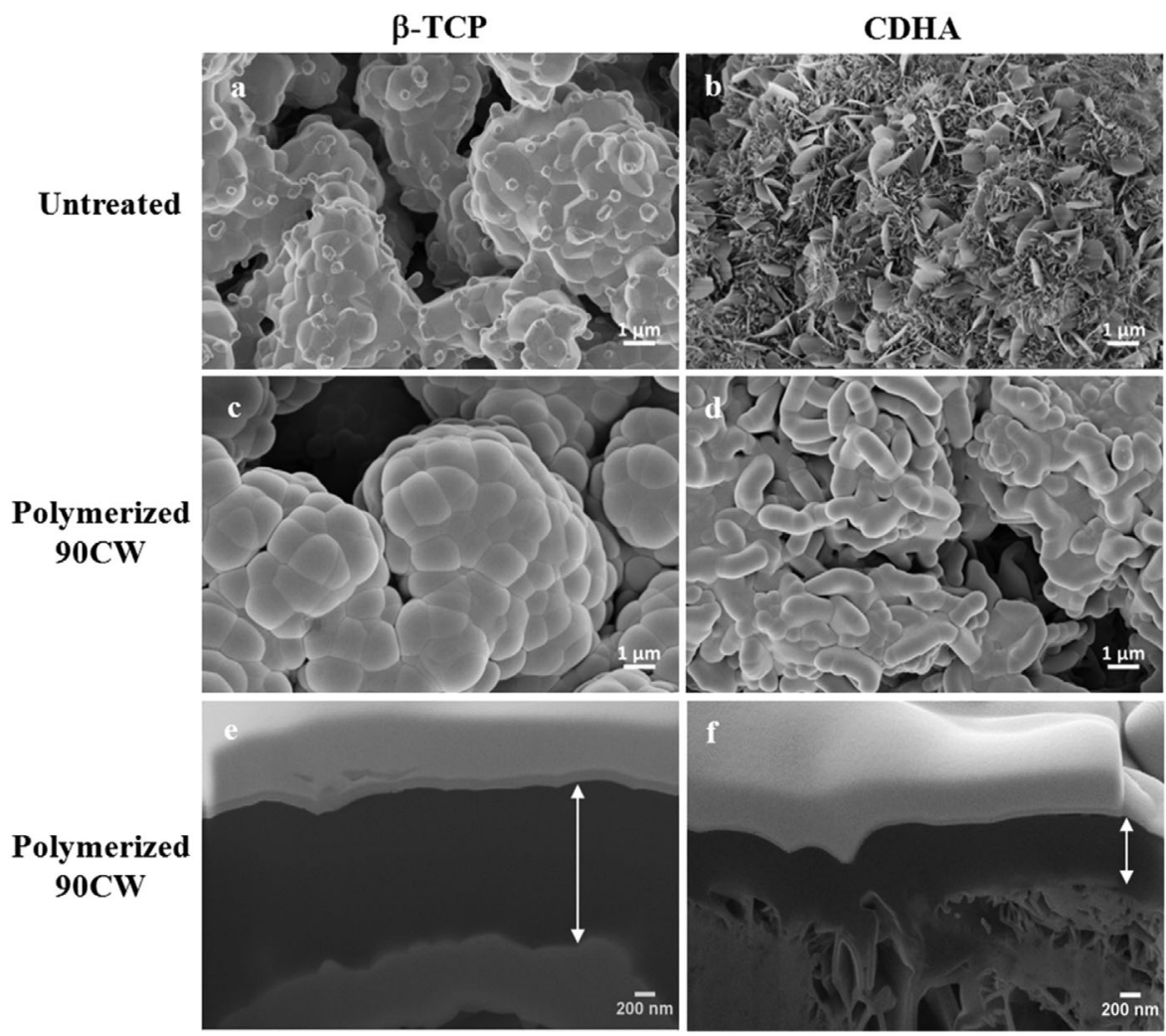

Figure 6. SEM images of the different materials before (top images) and after plasma polymerization with PCL-PEC (4:1). a) $\beta$-TCP, b) CDHA, c) $\beta$-TCP. $90 \mathrm{CW}$, and d) CDHA-90CW. FIB-SEM cross-section of the surface of both polymerized materials $\beta$-TCP-90CW e) and CDHA-90CW f). CW refers to continuous wave plasmas. Reproduced with permission from ref.[92] (C) 2016 Elsevier Ltd.

electrospun nanofibers loaded with a small molecule drug, ketoprofen, with $\mathrm{ALD} \mathrm{Al}_{2} \mathrm{O}_{3}$. The morphology and drug release were monitored as a function of the ALD $\mathrm{Al}_{2} \mathrm{O}_{3}$ thickness, ranging from 2 cycles to 200 cycles (Figure 7). The authors do not report a specific growth per cycle (GPC) for the ALD process. However, a thickness $<30 \mathrm{~nm}$ was sought. Remarkably, only 2 cycles of $\mathrm{ALD} \mathrm{Al}_{2} \mathrm{O}_{3}$ (corresponding approximately to $0.2-0.3 \mathrm{~nm}$ ) were found sufficient to reduce the ketoprofen release from $2 \mathrm{~min}$ (uncoated) to $24 \mathrm{~h}$ (coated with 2 cycle ALD layer). Increasing the thickness of the layer, a control over the release kinetics was achieved. The effect of the treatment on the PVA fibers and ketoprofen is also investigated. The melting point of the PVA fibers was found to decrease for the first 2 ALD cycles and then was found constant regardless the thickness of the ALD layer, indicating a surface interaction of the ALD layer with the PVA polymeric chains. However, the ketoprofen integrity was found to be retained upon the ALD treatment, as pointed out by UV spectroscopy of the released drug molecules.

Very recently, Kääriäinen et al. ${ }^{[106]}$ reported on a thorough investigation on the direct encapsulation of acetaminophen crystals by different ALD metal oxides, namely $\mathrm{Al}_{2} \mathrm{O}_{3}, \mathrm{TiO}_{2}$, and $\mathrm{ZnO}$. In this study, the effect of the ALD process on the drug morphology is studied, together with the delayed release and cytotoxicity of the ALD coatings. The ALD process was found not to significantly degrade acetaminophen crystals.
$\mathrm{TiO}_{2}$ deposited by means of $\mathrm{TiCl}_{4}$ was found to locally modify the crystal structure of the drug molecule, due to the formation of a binary system between $\mathrm{TiO}_{2}$ and acetaminophen. Furthermore, scanning electron microscopy (SEM) and energy dispersive spectroscopy (EDS) revealed a uniform ALD coating on the drug crystals, confirming the feasibility of this process also for the direct encapsulation of drugs. Finally, the cytotoxicity analysis showed the cyto-compatibility of the $\mathrm{TiO}_{2}$ layer, contrary to the $\mathrm{ZnO}$ layer which showed a higher cytotoxicity.

\subsubsection{Encapsulant-Controlled Drug Morphology}

Next to the control on the drug release and the possibility to tune the surface chemistry, encapsulation of drugs has been investigated for improving the stability of specific drug morphology. Considering the formulation of drug systems, poor solubility and low bioavailability often represent a hurdle, hindering their applicability. One of the approaches to overcome poor drug solubility is the use of amorphous solid state drugs. In the amorphous state, the higher accessible surface and the lack of lattice energies result in excess free energy. Consequently, the energy involved in the solubilization process is relatively low compared to crystalline systems. ${ }^{[109,110]}$ In order to keep the drug molecules in an amorphous state and 

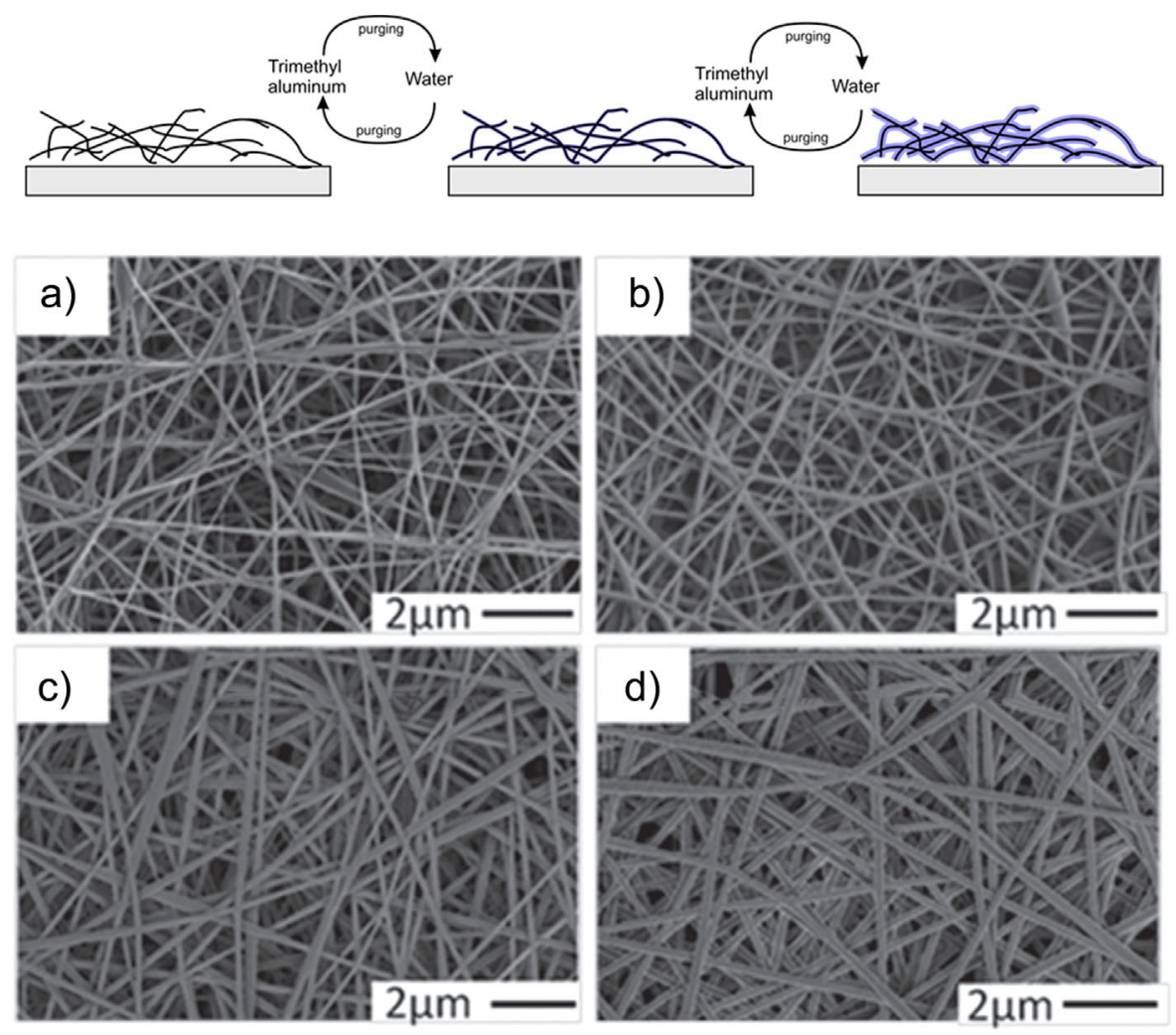

Figure 7. SEM micrographs of $7 \mathrm{wt} \%$ PVA electrospun mats coated with a) 0 cycles, b) 2 cycles, c) 20 cycles, and d) 200 cycles of $\mathrm{Al}_{2} \mathrm{O}_{3} \mathrm{ALD}_{\mathrm{L}}$ chemistry. On top, a schematic of the ALD process on the PVA fibers. Reproduced with permission from ref.[95] (c) 2015 WILEY-VCH Verlag GmbH \& Co. KGaA, Weinheim.

hinder recrystallization phenomena, minimization of molecule mobility and water vapor exposure need to be achieved. The use of barrier layers has been considered in order to delay the crystallization and, in turn, dry, single step methods like vapor phase processes represent a valid approach. In this regard, Bosselmann et al. ${ }^{[111]}$ performed pulsed PE-CVD on rapid frozen ketoprofen (RF-KET). Fluorinated coatings obtained from perfluorohexane-fed plasma were adopted in order to provide a physical and at the same time hydrophobic barrier coating capable of inhibiting crystallization of an amorphous drug/polymer system. The stability of the RF-KET upon plasma exposure is confirmed by the absence of degraded drug molecules in high performance liquid chromatography (HPLC). Moreover, the morphology of the drug molecule in its amorphous state was preserved during the pulsed PE-CVD process, as shown by differential scanning calorimetry (DSC). The amorphous drug molecules coated with the hydrophobic plasma polymer were shown to keep their amorphous morphology up to 6 months after encapsulation when stored at a temperature close to the drug molecule glass temperature and in high relative humidity. The uncoated RF-KET showed instead crystallization after 3 days. Despite the excellent barrier properties shown by the hydrophobic coating, drug release experiments showed a poor control over the out-diffusion of RF-KET. The authors attributed this behavior to the poor adhesion of the fluorinated polymer on the hydrophilic drug surface.
Encapsulation of amorphous drugs has been investigated in details also with iCVD polymers by Christian et al. ${ }^{[76,78]}$ Three different polymer compositions were deposited by iCVD on top of amorphous clotrimazole films to study the stability of the drug solid state: two different stimuli-responsive polymers, poly(2hydroxyethyl methacrylate) [p-HEMA], hydrogel, and poly-(methacrylic acid) [p-MAA], pH-responsive, and a non-responsive hydrophobic fluorinated polymer, poly(perfluorodecyl acrylate) $[\mathrm{p}-\mathrm{PFDA}]{ }^{[76]}$ In their study, the authors compare the effect of different polymer chemistries on the stability of the amorphous clotrimazole by means of optical microscopy and X-ray diffraction (Figure 8a). Storage of the samples at ambient temperature, 50 and $70^{\circ} \mathrm{C}$ showed that hydrophilic polymers such as p-HEMA and pMAA are able to keep the amorphous state of the drug. p-PFDA, instead, due to its hydrophobic nature and intrinsic crystallinity, ${ }^{[112]}$ showed complete crystallization of clotrimazol already at $50^{\circ} \mathrm{C}$. In-situ X-ray diffraction as a function of temperature confirmed these results, showing that p-HEMA and p-MAA prevent clotrimazole crystallization through quick heat treatments up to the melting point of the crystalline drug (Figure 8b). Finally, wrinkle formation upon the deposition of the iCVD polymers has been reported in this work, due to differences in elastic behavior of the drug layer and the polymer. An extended study has been reported in a follow-up work (Figure 8c). ${ }^{[78]}$ Such wrinkles are very promising as these might allow the construction of surface layers of significantly larger surface area, which often helps faster drug release. 
a)

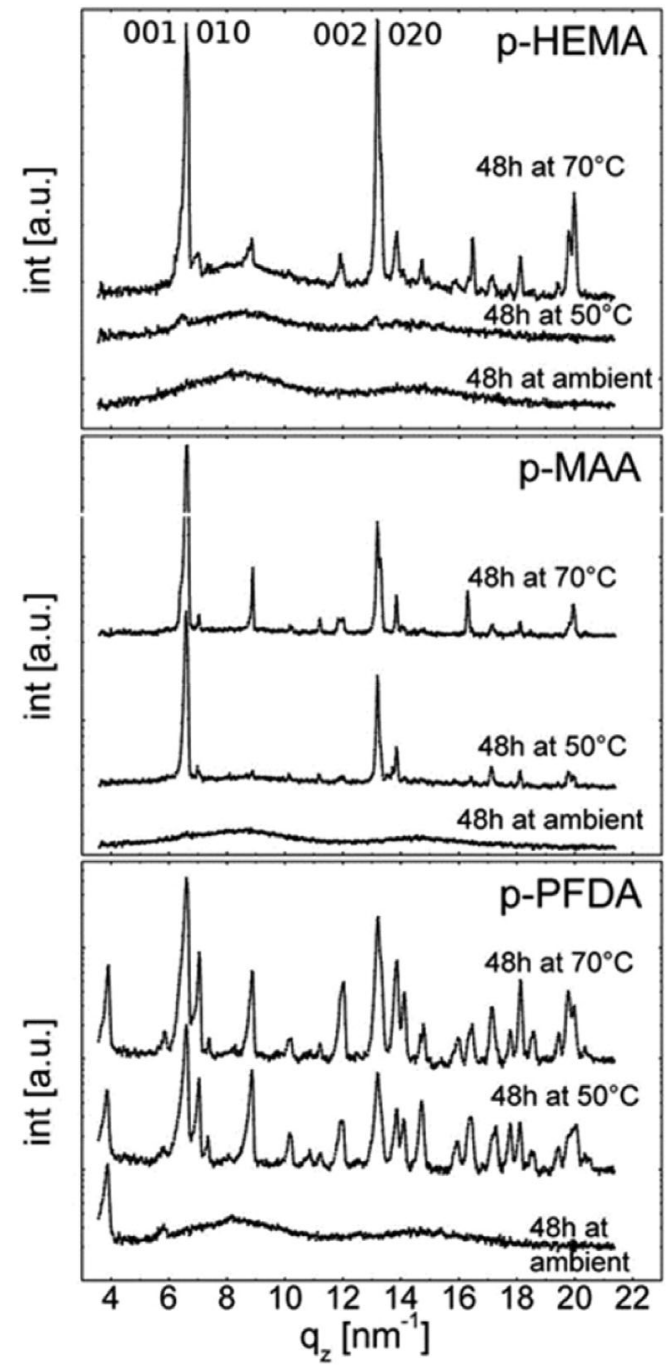

b)
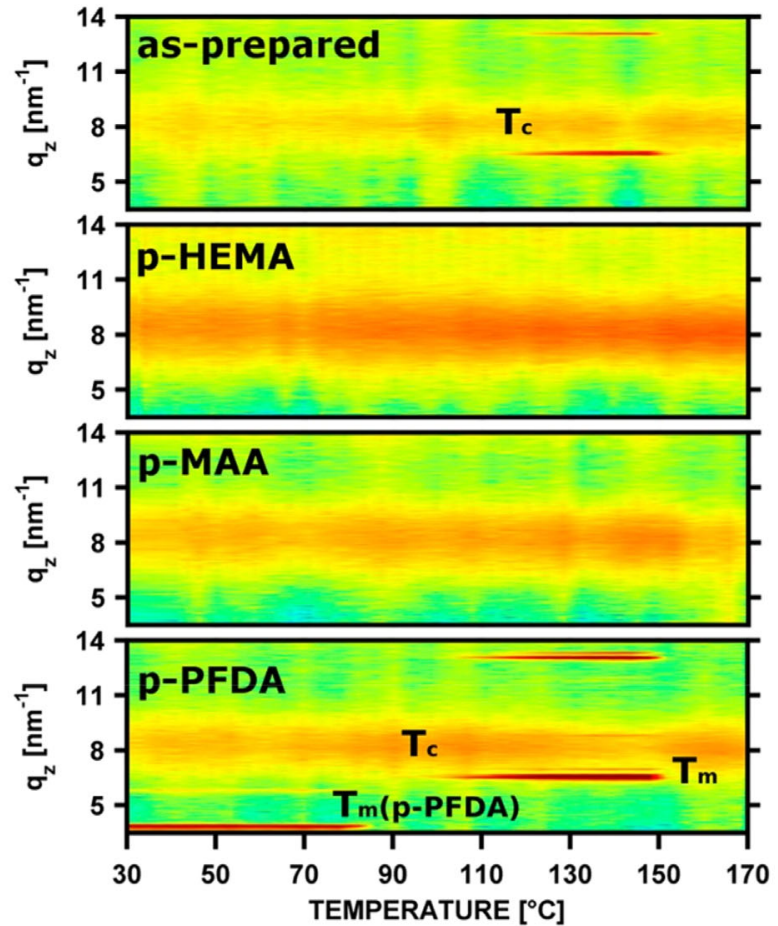

C)

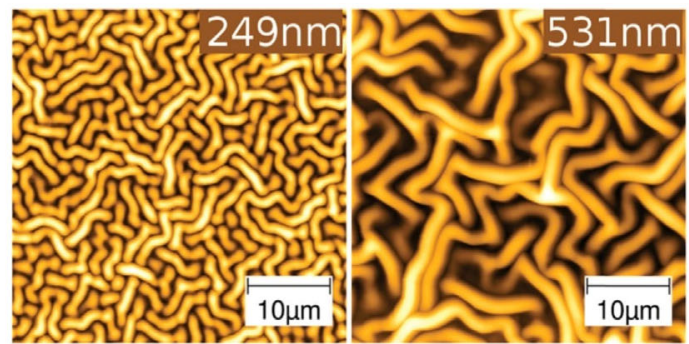

Figure 8. a) X-ray diffraction scans of clotrimazole encapsulated with different polymers (p-HEMA, p-MAA, p-PFDA) after storage at different conditions; b) In-situ X-ray diffraction scans of amorphous clotrimazole films, encapsulated by iCVD layers, at different temperatures. Reproduced with permission from ref.[64] (c) 2016 American Chemical Society. c) AFM height images of pHEMA films coated on top of clotrimazole spacing layers of varying thicknesses, as reported in the labels. Reproduced with permission from ref.[66] Published by The Royal Society of Chemistry.

\subsection{Embedding}

As afore-mentioned, solubility and bioavailability of drug molecules represent a major challenge for their use in medication. One possible strategy, next to the encapsulation, is the formation of the so-called solid molecular dispersions or solid solutions. In this approach, the drug is dispersed in an (usually hydrophilic) inert solid matrix in order to enhance the drug solubility during application. Additionally, control over the dissolution rate can be achieved by choosing a specific chemistry or cross-linking degree. Numerous studies on solid dispersions have been published and showed the advantageous characteristics of solid dispersions in improving the solubility and dissolution rate of poorly water-soluble drugs. However, long-term stability problems, for example, drug crystallization and decrease in dissolution rate, have been reported, due to the complexity of the methodology used to obtain the solid dispersions. Detailed insights into the subject can be found in the literature. ${ }^{[113,114]}$ Vapor-phase methods have been recently adopted for the engineering of solid dispersion, either in single-step processes ${ }^{[115-117]}$ or for the further stabilization of drug-matrix dispersions. ${ }^{[108]}$ The literature mostly reports on strategies for active biological compounds added as a second step to vapor-phase deposited substrates. This second step is often carried out through the adsorption of the drug/bio-active material in solution processes. $^{[118]}$

A novel method for the inclusion of drug molecules has been demonstrated by Palumbo et al. ${ }^{[116]}$ A one-step spray-assisted atmospheric- pressure plasma process for the synthesis of solid dispersions is investigated. Lysozyme (Lyz) dispersed in water is sprayed in the plasma reactor chamber together with a helium flow. Ethylene-fed plasma is used in order to deposit a hydrocarbon matrix. Water-droplet encapsulated Lyz molecules 
enter into the plasma region, during which the vinyl bonds of ethylene monomers begin to dissociate, polymerizing into polyethylene-like (PE) coating. At the same time, Lyz molecules start to deposit intact on the substrate, due to the protective action of the water droplets. The droplets dissociate and vaporize due to discharge and gas heating, allowing the embedding of Lyz into the polymer matrix. ${ }^{[117]}$ The amount of embedded Lyz was controlled by process parameters, as clearly evidenced by IR spectra in Figure 9a.

The retention of the Lyz structure is a crucial parameter for the assessment of the deposition methodology. MALDI-TOF (Figure 9b) and HPLC were used for the determination of the embedded and released Lyz, respectively. The two analyses point out that the majority of embedded Lyz retained its structure. Broadening of the peaks indicated minor changes in the embedded molecule structure. Nevertheless, its activity is maintained, as pointed out by an Agar diffusion assay.

With a similar approach, Vancomycin was embedded in a PE matrix.${ }^{[119]}$ Remarkably, the polymerized layers were comprised of spherical features with nanometric size (Figure 10a). In order to get further insights into the nanocapsules's structure, vancomycin was replaced with fluorescein in the aerosol solution and analyzed with confocal microscopy (Figure 10b).

From the images, spherical structures, hundreds of nanometers wide, have been identified consisting of a hydrocarbon polymeric shell and a vancomycin/fluorescein containing core. By varying the plasma parameters, it is possible to tune the dimensions and the aggregation of the nanocapsules. These structures seem to release the contents of their cores after immersion in water. $^{[119]}$
Modification of a pre-loaded polymer is an alternative route for the embedding of active molecules. ALD has been adopted for the synthesis of hybrid organic-inorganic blends containing the model molecule fluorescein. ${ }^{[108]}$ Films of PEG 400, a liquid polymer at room temperature, were converted into a solid hybridized film by means of ALD. ALD zinc oxide was deposited on top of fluorescein-loaded PEG and found able to penetrate deep into the polymer (Figure 11). ZnO was formed within the bulk of this gel like film. While PEG works as loading medium, the subsequent ALD process converts the liquid drug-solution into a solid hybrid layer, forming the storage phase. A release layer (poly(3,4-ethylenedioxythiophene), PEDOT) is added on top of the PEG-ZnO, serving as gate keeper, for the engineering of a releasing device, as it will be discussed further in the review.

\subsection{Immobilization/Surface Modification}

Vapor-phase methods are also adopted for the deposition of buffer layers and subsequent (covalent) immobilization of drug molecules or, when plasma based techniques are used, these techniques are applied for the surface modification of polymeric matrices adopted as substrates.

Myung et al. ${ }^{[120]}$ reported on the immobilization of doxorubicin on the surface of plasma polymerized acrylic acid. The presence of carboxylic groups retained during plasma polymerization enhances the loading of the drug molecule and allows, already without encapsulation layer, a slower release of the drug. In a similar fashion, enhanced drug loading can be achieved by modifying the polarity of the surface, either with

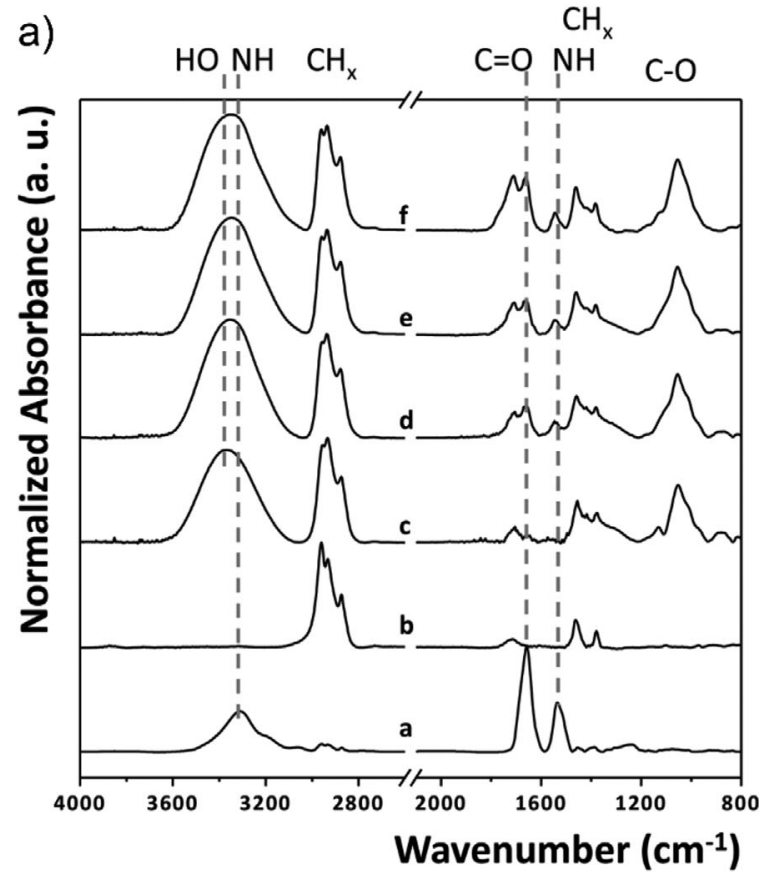

b)

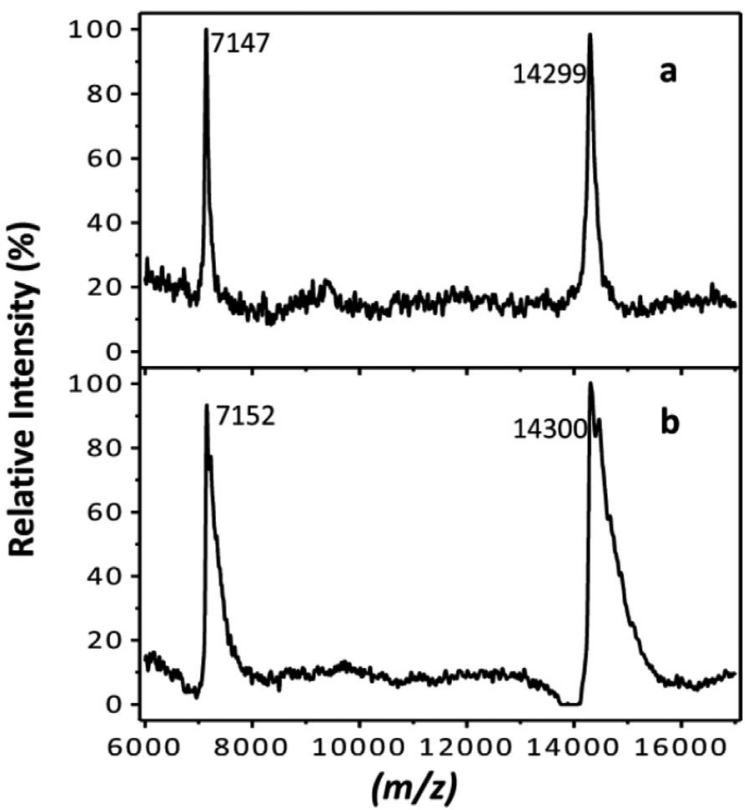

Figure 9. a) FTIR spectra of: casted Lyz (a); films deposited at an atomizer flow rate of 0 (b), 3 (c), 4 (d), 5 slm (e) with a $5 \mathrm{mg} \mathrm{mL}^{-1}$ Lyz solution; and at 5 $\mathrm{s} / \mathrm{m}$ atomizer flow rate, but with a $8 \mathrm{mg} \mathrm{mL}^{-1}$ Lyz solution (f). b) MALDI-TOF mass spectra of native lysozyme (a) and the HiLyz sample (b). Reproduced with permission from ref.[104] (c) 2015 WILEY-VCH Verlag GmbH \& Co. KGaA, Weinheim. 
a)

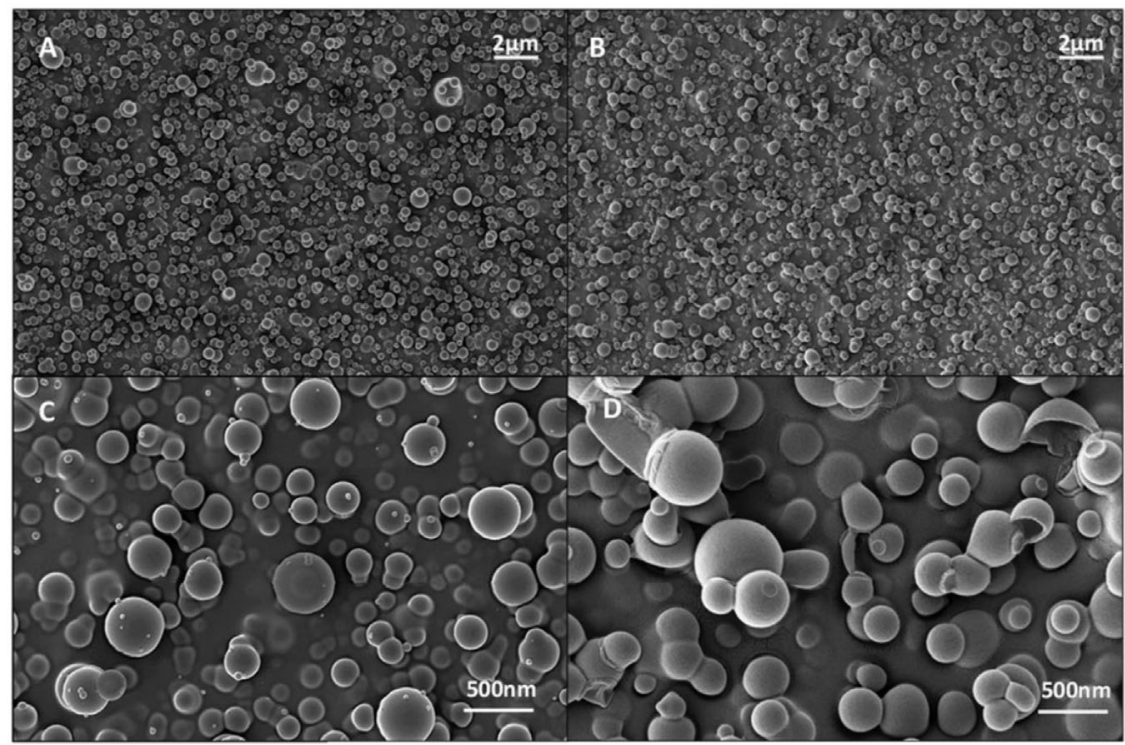

b)

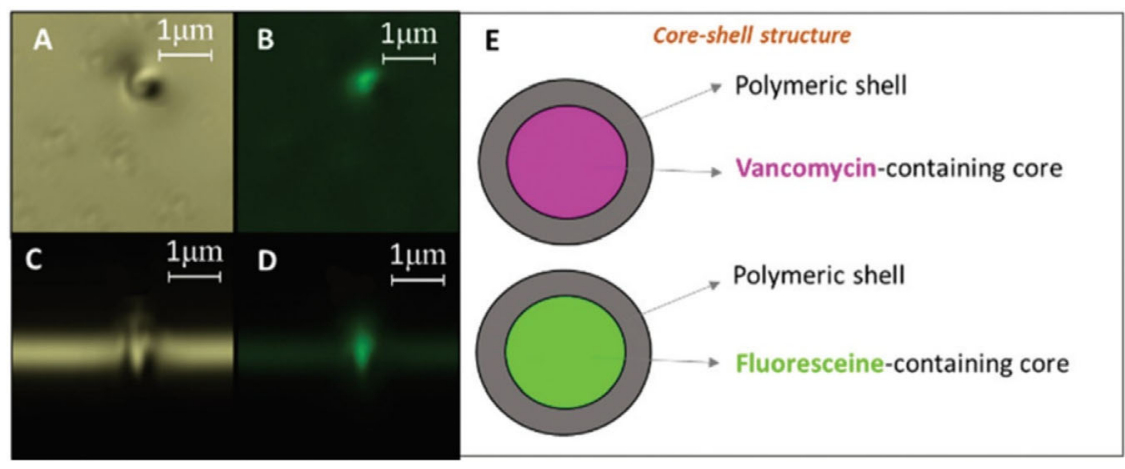

Figure 10. a) Top view SEM images (extraction voltage of $2 \mathrm{kV}$ ) of the continuous wave (CW) sample before immersion at $10 \mathrm{k} \times(\mathrm{A})$ and $50 \mathrm{k} \times(\mathrm{C})$, and after $60 \mathrm{~min}$ of immersion in water at $10 \mathrm{k} \times(\mathrm{B})$ and $50 \mathrm{k} \times(\mathrm{D})$. b) Confocal microscopy images: top view reflection (A) and top view fluorescence (B), section reflection (C) and section fluorescence (D) of the nano-capsules deposited in CW mode, feeding the aerosol with fluorescein. A scheme of the hypothesized core-shell structure for vancomycin and fluorescein containing nanocapsules is shown (E). Reproduced with permission from ref.[107] This journal is (c) The Royal Society of Chemistry 2017.

plasma polymerization or with grafting/surface modification of samples. As afore-mentioned, Bahtt et al. ${ }^{[9]}$ adopted this approach to homogenize the loading of the drug molecules on the substrate and increase the amount loaded. Song et al. ${ }^{[121]}$ reported on the surface modification of bare metal stents by means of PE-CVD. $\mathrm{TiO}_{2}$ layers were obtained by plasma treatment of titanium(IV) tetraisopropoxide (TTIP) and subsequently further functionalized by water-fed plasma. This last step introduced hydroxyl groups on the $\mathrm{TiO}_{2}$ surface, allowing the grafting of drug molecules. $\alpha$-lipoic acid (ALA), heparin, and abciximab were successfully immobilized on the stent surface.

Labay et al. ${ }^{[101]}$ investigated the effect of atmospheric plasma treatment of PP meshes, as mentioned in the previous paragraph. The plasma treatment was found to drastically modify the morphology and surface chemistry of the meshes. Hydrophilic surfaces (contact angle 20 ) and high surface roughness were measured after plasma treatment (Figure 12).
Upon loading of the PP meshes with ampicillin, a significant increase in the loaded amount when compared to untreated samples was reported. Short treatment times showed a 2-fold increase which can be attributed to the improved surface wettability, while longer times loaded up to 3-fold the weight percentage obtained by the untreated PP. The higher loading at higher plasma treatment times was attributed to the additional grafting of new $\mathrm{C}-\mathrm{O}$ bonds and the major available surface area given by the higher roughness.

Canal et al. ${ }^{[122]}$ evaluated the surface modification of microporous $\beta$-TCP scaffold ceramics with He-fed atmospheric pressure plasma jet (APPJ) for the design of controlled release matrices. The plasma treated microporous $\beta$-TCP ceramics have been loaded with doxycycline hyclate. The authors show that the treatment with the atmospheric plasma needle using He led to a progressive increase in oxygen content with longer treatment times. Moreover, charging of the surface develops, influencing the loading of the drug molecules. It is showed that the 
a)

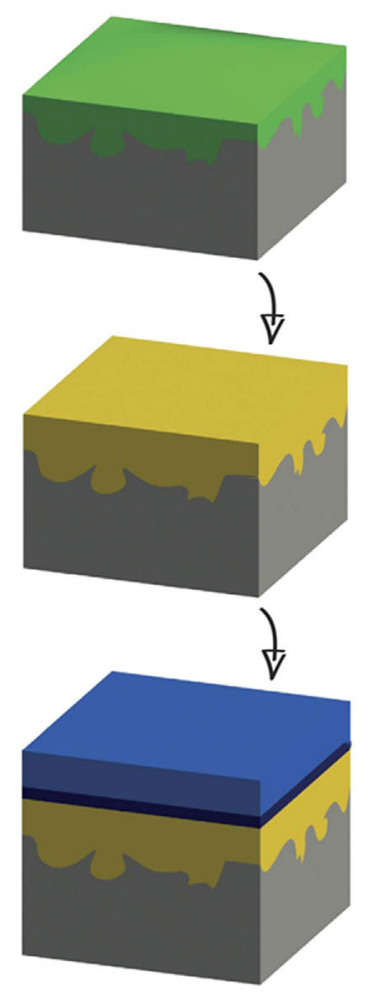

b)

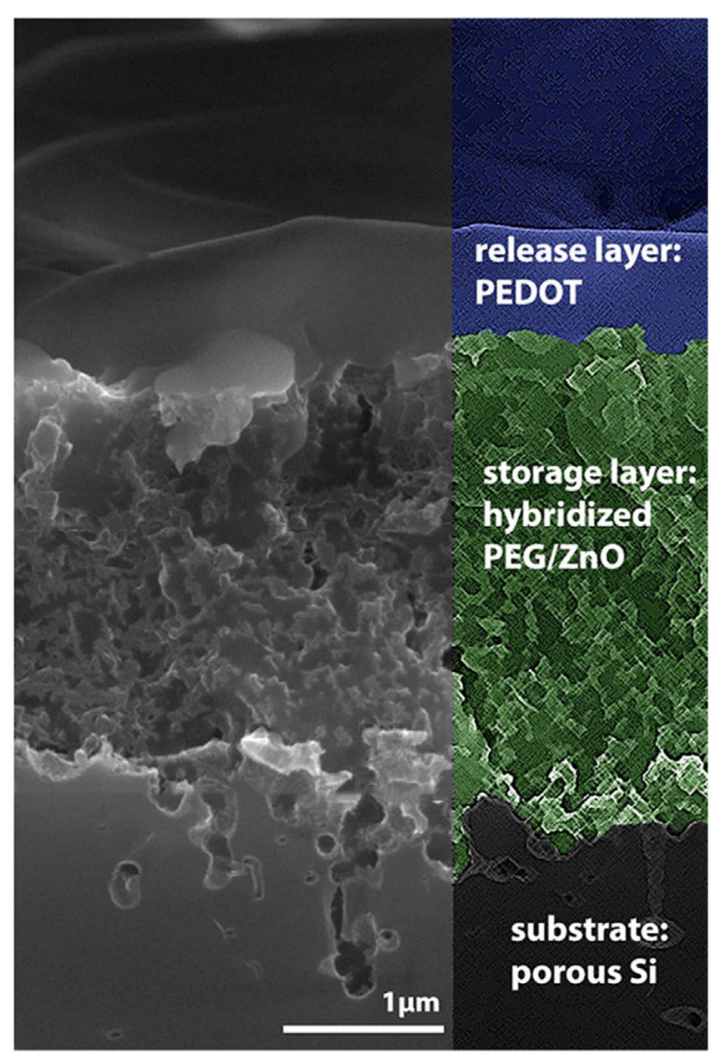

Figure 11. a) Spin-coating of a drug-containing PEG film (green) onto the substrate, followed by the formation of a hybrid structure by means of ZnOALD in "yellow". Last, coating with conducting polymer layers (blue). b) Cross-sectional Scanning Electron Microscopy (SEM) image of the PEG/ZnO release system. The hybridized storage layer is marked in green (middle) with the primary conducting polymer layer on top, represented in blue. The process is schematized on the left side of the image. Reproduced with permission from ref.[96] Copyright (C) 2016, Rights Managed by Nature Publishing Group.

hydrophilic surface and charge developed are able to slow down the release of the loaded drug, achieving more than $33 \%$ improvement in retaining drug release for optimized APPJ treated $\beta$-TCP with respect to untreated samples.

Finally, plasma treatment can be used to induce higher crosslinking degree on pre-loaded polymers. In a series of studies on the effect of inert gas fed-plasma treatment on polymer structure, Yamahuci et al. ${ }^{[123-125]}$ investigated the treatment of polymer powders ${ }^{[123]}$ or direct treatment of preloaded drugpolymer tablets ${ }^{[124,125]}$ Cross-linking of the polymers' outer shell backbone leads to a pseudo-encapsulation effect, decreasing the drug release rate. Similar conclusions have been achieved by Hagiwara et al. ${ }^{[26]}$ in treating curcuminloaded poly(ethylene-co-vinyl acetate) with different plasma environments $\left(\mathrm{O}_{2}, \mathrm{~N}_{2}\right.$, Ar).

\section{Conclusions and Outlook to Future Device Fabrication}

Drug delivery, targeted to a specific body location and/or in time, is an interesting feature of the new generation biomedical devices and it can be achieved through the use of "smart" formulation embedded in tunable polymer shells that hinder the drug release until a proper stimulus is detected ${ }^{[127]}$ Stimuli-responsive polymers serve this purpose very well: they undergo changes in thickness, mesh size and/or surface energy depending on the external stimuli, like temperature, ${ }^{[128]} \mathrm{pH}^{[66,64]}$ and light. ${ }^{[52,129]}$ Summarizing the previous paragraphs, the deposition of this kind of polymers from the vapor phase can have several advantages: (i) the absolute lack of solvents or high temperature allows easy drug encapsulation, without inducing damages that would harm the formulation or components therein and later the therapeutic effects; (ii) the absence of plasticizers, leachable, and surfactants eliminates the health considerations associated with these components; (iii) the possibility to reproduce interesting geometries by conformally coating the tridimensional features of a substrate; (iv) the easy tunability of the film and surface properties. Surface modifications that yield a large density of functional groups can be used to tether biomolecules (e.g., proteins, DNA fragments) to drug delivery devices in order to detect biological entities like bacteria and viruses and have very specific therapeutic actions. ${ }^{[130]}$ In the previous sections, different strategies were presented for loading a drug within/beneath a polymer deposited from the vapor phase. These drug-loaded polymers were then successfully integrated in several delivery devices for therapeutic dosing. In the next section, vapor deposited polymers are presented together with some examples of devices where the 

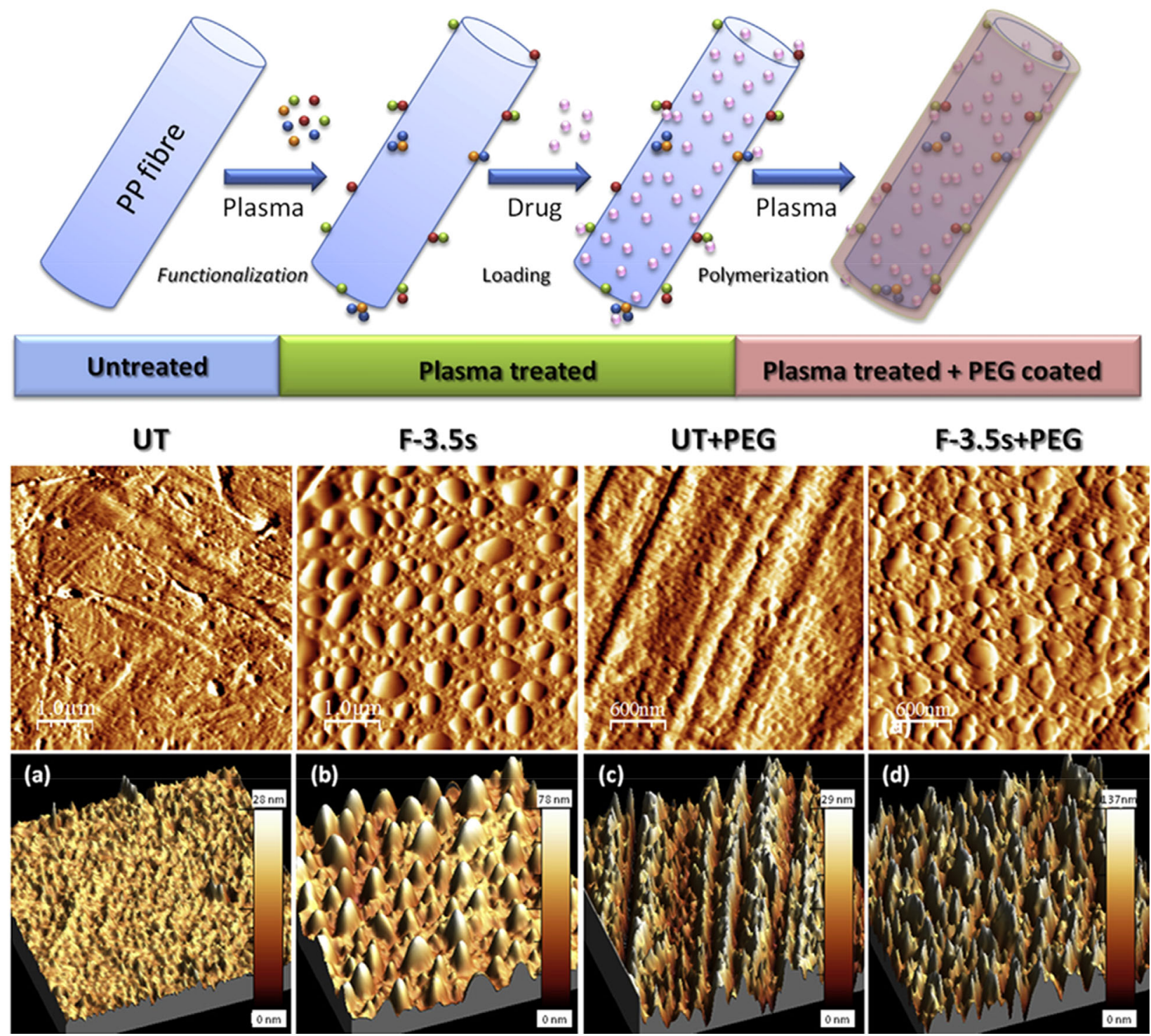

Figure 12. PP fiber modified by a first plasma treatment aimed at increasing the adhesion of drug molecules, followed by a second plasma deposition of a PEG polymer. The process is schematized on top of the figure. AFM topography (top images) of the untreated (UT) a), air-plasma treated for $3.5 \mathrm{~s}$ (F-3.5 s) plasma-treated b) PP meshes, and after PEG plasma coating: UT + PEG c) and F-3.5 s + PEG d), with the respective 3D reconstitutions of the fiber surface (bottom images). Reproduced with permission from ref.[89] (C) 2015 Elsevier Ltd.

use of advanced growth methods could be highly beneficial for the future generation.

Devices based on stimuli-responsive polymers. Burst release of fluorescein was demonstrated by a device made of coaxial nanotubes consisting of a shape-memory shell and a hydrogel core ${ }^{[131]}$ The swollen hydrogel was loaded with the dye and dried to deswell. At high temperature, the shape-memory shell shrank, squeezing the dye out of the structure. Both the hydrogel core and the shell were deposited by iCVD, by sequential depositions of the two polymers into the pores of an anodized aluminum oxide membrane. Thanks to the conformality of the iCVD process, coaxial structures at the nanoscale were obtained, resulting in an effective burst release. Fluorescein was used as a model molecule in this study, for easy monitoring by optical spectroscopy. Nevertheless, the same principle could be used for burst release of drugs upon the correct stimuli.

Stimuli-responsive polymers were also used to regulate the flow of drugs through microfluidic devices. ${ }^{[132]}$ Baldi et al. showed a microfluidic system made of a combination of hard and soft materials. In this case, a glucose-sensitive hydrogel controls the flow of insulin in response to changes in glucose concentrations. ${ }^{[133]}$ When the hydrogel expands, upon changes in glucose concentration, it closes down the flow inlet so insulin cannot be delivered.

Also light can be used as external stimulus to trigger delivery, for example, on skin patches. Light-responsive polymers, as the one synthetized by Pauly et al. ${ }^{[129]}$ have chromoforic groups like spiropyran tethered to the polymer backbone. The light induces a 
reversible hydrolysis in the spiropyran moieties, changing the wettability and the permeability of the polymer. An enhanced flow of caffeine was registered through the polymer membrane when this was exposed to light.

Devices based on electrochemical stimuli. Next to responsive systems, also devices based on electrochemical triggering have been shown to have several interesting features especially for transdermal delivery. Boehler et al. showed an example of a promising device in which the drug delivery, upon electrical stimulus, was coupled with enhanced drug storage within a hybrid material made of $\mathrm{ZnO}$ and polyethylene glycol. ${ }^{[108]}$ As described in the previous section, the polymer served as dispensing medium for the liquid drug, which was then solidified by deposition of ALD ZnO. This hybrid layer could store up to $25 \mathrm{ng}$ of drug in a few micron thicknesses. The delivery was achieved by applying a voltage to the conductive polymer that was added on top of the hybrid structure. Negative bias triggered the release while positive bias suppressed it.

He et al. ${ }^{[134]}$ showed another example of electrochemically triggered drug delivery interfaces based on a flexible electrode consisting of thin gold films, deposited onto Kapton, coated with doxorubicin-loaded reduced graphene oxide. The release in this system was driven by a positive potential bias that decreased the $\mathrm{pH}$ close to the electrode surface and enhanced the desorption of the drug.

Devices that deliver drugs with a spatiotemporal resolution upon stimuli can pave the way toward future biochemically regulated therapeutics reservoirs or "portable pharmacies". An early example of such device is shown in Figure 13a. It is a neurotransmitter delivery device with individually controlled delivery points. ${ }^{[135]}$ By applying local pulses, the neurotransmitter acetylcholine was delivered independently from the different delivery points with a temporal resolution of $50 \mathrm{~ms}$ from the time of voltage on to the delivery.

Multilayer devices. Steady delivery over extended period of time can be important for therapeutic dosing of the drug. Dowling et al. demonstrated a drug eluting system based on a multilayer in which the drug was sandwiched between two plasma polymers. ${ }^{[98]}$ The role of the upper layer was to restrict the elution rate, resulting in a significantly retarded release over a 7 days period. Another multilayer device was demonstrated by Bhatt et al. ${ }^{[99]}$ In this case, the drug was sandwiched between a cell-repellent top layer and a diffusion barrier layer that would regulate the drug elution. The cell-repellent layer promoted uninterrupted drug release.

Devices for transdermal delivery. Strategies for transdermal drug delivery are currently under investigation to expand the library of drugs that can be delivered through the skin. Currently, due to the barrier properties of the human skin only very small and lipophilic drugs can be delivered through external patches. Microneedles that puncture only the most superficial layer of the skin would be a pain-free solution that would allow the delivery of many more pharmaceutics. ${ }^{[136]}$ Overlaying a coating from the vapor phase that would keep the microneedle shape but add to the needle material some interesting surface properties (e.g., functional groups for biomolecule immobilization) would augment the functionalities achievable by such delivery systems. An early example of surface modification of microneedles was shown by Nair et al. ${ }^{[137]}$ They treated the surface of plastic microneedles with an oxygen plasma to enhance the wettability of the needle surface toward different drug formulations. As demonstrated here, depositing thicker layers made from polymers even would allow introducing additional functionality from which this very application would definitely benefit, for example, hydrogel formation on contact with skin - fluid would weaken the mechanical strength so that pain sensation would minimize.

Next to the implementation of existing encapsulating solutions into working and 'smart' devices, new deposition methods could be used in order to encapsulate/embed drug molecules into organic or inorganic matrix. As mentioned in Section 2, molecular layer deposition is, next to ALD, a selflimiting layer-by-layer vapor phase deposition method that allows the deposition of fully organic or hybrid organic-inorganic a)

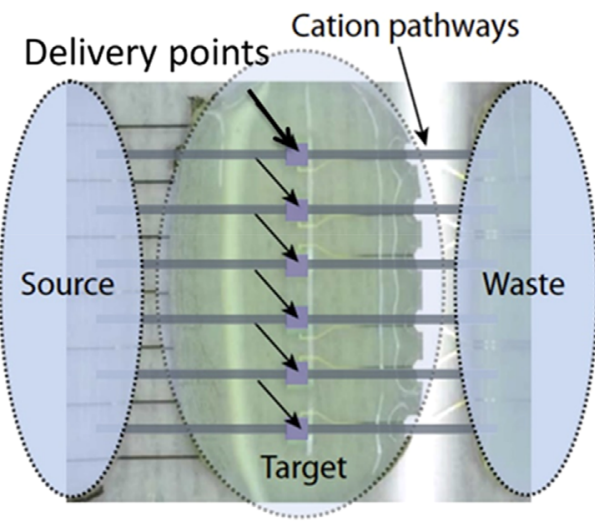

b)

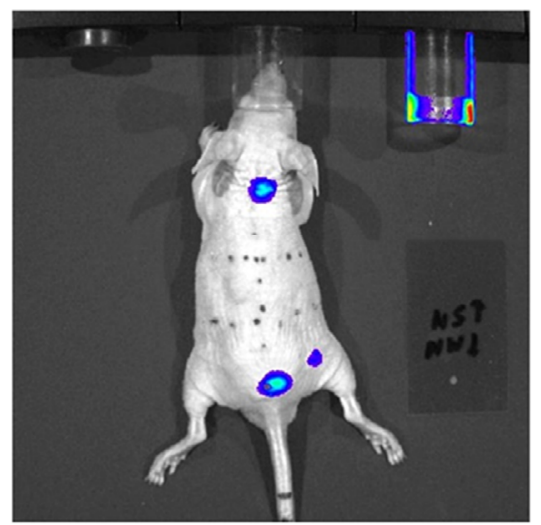

Figure 13. a) Top-view of an electrically controlled chemical delivery circuit, where charged compounds can be released independently from several delivery points within tens of milliseconds. Reproduced with permission from ref.[123] Copyright (C) 2016, The Authors. b) First in-vivo test of an injected glucose sensor encapsulated in a pHEMA/EDGA shell deposited by iCVD. Reproduced from Reproduced with permission from ref.[135] Copyright (C) 2011, The Authors. 
materials. The range of polymers that can be deposited by MLD has rapidly broadened in the last years, together with the different functionalities that can be implemented. Polyamine, polyurea and polyesters are only few examples of the classes of polymers that can be synthesized with this method. Furthermore, a new class of materials, so called 'metalcones', hybrid organometallic polymers, represents an additional possibility. In the literature, complete reviews summarizing the different possible polymers are available. ${ }^{[57,138,139]}$ Although still no examples of encapsulated drug are present in the literature by MLD deposited polymers, the potential impact of this technique in the field is remarkable. MLD thin films have already been applied as organic interlayer in moisture permeation barrier, ${ }^{[140]}$ Cu-barrier diffusion layers, ${ }^{[141]}$ photoactive passivation layers, ${ }^{[142]}$ and as stabilizers during metallic nano-particles in reforming conditions. ${ }^{[143]}$ Moreover, sharing the same conformality and sub-nm thickness control shown by ALD, also the coating of complex tridimensional structures has been demonstrated, together with the possibility to change the surface chemistry to improve wettability and stability. ${ }^{[144]}$

One of the future challenges that can be envisioned for vapor-deposited polymers is to surpass all the regulations that would allow their use in human body. Indeed, when the polymer shell is non-toxic and biodegradable and the degradation products are biocompatible and pharmacologically inactive, the targeted delivery systems can be directly injected into the body. An injectable hydrogel was proposed by Jeong et al. made of blocks of poly(ethyleneoxide) and poly(L-lactic acid). ${ }^{[145]}$ In this case, the bioactive molecules entrapped in the polymer matrix was released in the body at first by diffusion, and later by the combination of both diffusion and degradation mechanisms. Similarly, a biodegradable hydrogel was functionalized with protoporphyrin as a fluorescence tag to track the drug delivery in-vivo by multispectral fluorescence imaging in nude mice. ${ }^{[146]}$ Preliminary in-vivo tests of vapor-deposited polymers for drug delivery already exist (e.g., an optode sensor was encapsulated in a pHEMA-EGDMA shell by iCVD and injected into rats, ${ }^{[147]}$ as reported in Figure $13 \mathrm{~b}$, but more demonstrations are required for the significant implementation of vapor deposited polymers in the next generation of biomedical devices.

In summary, the deposition of polymers from the vapor phase offers versatile strategies for targeted drug delivery, such as the absence of solvents, the low process temperature, the possibility to tailor the physical-chemical properties of the processed layer in a single synthetic route, and of combining different processes to achieve multi-layered and, in turn, multi-functional polymers, overcoming some of the drawbacks of classical encapsulation/ embedding methodologies. Continued implementation of these polymers in future devices will have a significant impact on the next generation of therapies.

\section{Acknowledgements}

BioTechMed, Graz, is gratefully acknowledged. This project has received partial funding from the European Research Council (ERC) under the European Union's Horizon 2020 research and innovation programme (grant agreement 715403).

\section{Conflict of Interest}

The authors declare no conflict of interest.

\section{Keywords}

ALD, drug encapsulation, embedding, hydrogel, iCVD, PE-CVD, surface modification

Received: July 28, 2017

Revised: September 1, 2017

Published online:

[1] N. D. Volkow, G. J. Wang, M. W. Fischman, R. Foltin J. S. Fowler, D. Franceschi, M. Franceschi, J. Logan, S. J. Gatley, C. Wong, Y. S. Ding, R. Hitzemann, N. Pappas, Life Sci. 2000, 67, 1507.

[2] S. Grassin-Delyle, A. Buenestado, E. Naline, C. Faisy, S. BlouquitLaye, L. J. Couderc, M. Le Guen, M. Fischler, P. Devillier, Pharmacol. Ther. 2012, 134, 366

[3] L. Z. Benet, J. Pharmacokinet. Biopharm. 1978, 6, 559.

[4] M. Kalani, R. Yunus, Int. J. Nanomed. 2011, 6, 1429.

[5] X. Xu, M. A. Khan, D. J. Burgess, Int. J. Pharm. 2011, 419, 52.

[6] W. Abdelwahed, G. Degobert, S. Stainmesse, H. Fessi, Adv. Drug Deliv. Rev. 2006, 58, 1688.

[7] L. X. Yu, Pharm. Res. 2008, 25, 781.

[8] T. Loftsson, M. E. Brewster, J. Pharm. Sci. 1996, 85, 1017.

[9] A. T. M. Serajuddln, J. Pharm. Sci. 1999, 88, 1058.

[10] A. C. Williams, B. W. Barry, Adv. Drug Delivery Rev. 2004, 56, 603.

[11] Y. Sudhakar, K. Kuotsu, A. K. Bandyopadhyay, J. Controlled Release 2006, 114, 15

[12] S. H. Freiesleben, J. Soelberg, A. K. Jäger, J. Ethnopharmacol. 2015 174, 561.

[13] A. N. Shikov, O. N. Pozharitskaya, V. G. Makarov, H. Wagner, R. Verpoorte, M. Heinrich, J. Ethnopharmacol. 2014, 154, 481.

[14] H. Park, K. Park, in Polymers of Biological and Biomedical Significance. ACS Symposium Series; American Chemical Society, Washington, DC 1993, p. 2.

[15] D. Jones, Pharmaceutical Applications of Polymers for Drug Delivery, 2004.

[16] N. P. S. Chauhan, A. K. Pathak, K. Bhanat, R. Ameta, M. K. Rawal, P. B. Punjabi, in Encyclopedia of Biomedical Polymers and Polymeric Biomaterials. (Ed: Munmaya Mishra), CRC Press 2015, p. 5929.

[17] J. K. Jackson, K. Letchford, B. Z. Wasserman, L. Ye, W. Y. Hamad, H. M. Burt, Int. J. Nanomed. 2011, 6, 321.

[18] S. Schrank, B. Kann, E. Saurugger, H. Ehmann, O. Werzer, M. Windbergs, B. J. Glasser, A. Zimmer, J. Khinast, E. Roblegg, Mol. Pharm. 2014, 11, 599.

[19] K. Ulbrich, K. Holá, V. Šubr, A. Bakandritsos, J. Tuček, R. Zbořil, Chem. Rev. 2016, 116, 5338.

[20] K. Ariga, Q. Ji, M. J. McShane, Y. M. Lvov, A. Vinu, J. P. Hill, Chem. Mater. 2012, 24, 728.

[21] H. Chen, D. Liu, Z. Guo, Chem. Lett. 2016, 45, 242.

[22] J. D. Rocca, D. Liu, W. Lin, Acc. Chem. Res. 2011, 44, 957.

[23] S. Taherzade, J. Soleimannejad, A. Tarlani, Nanomaterials 2017, 7, 215.

[24] R. G. Strickley, Pharm. Res. 2004, 21, 201.

[25] E. Bouyer, G. Mekhloufi, V. Rosilio, J. L. Grossiord, F. Agnely, Int. J. Pharm. 2012, 436, 359.

[26] P. Huang, H. Song, Y. Zhang, J. Liu, J. Zhang, W. Wang, J. Liu, C. Li, D. Kong, ACS Appl. Mater. Interfaces 2016, 8, 29323. 
[27] M. Zayed, C. Tourne-Peteilh, M. Ramonda, G. Rethore, P. Weiss J. Martinez, G. Subra, A. Mehdi, J.-M. Devoisselle, P. Legrand, Int. J. Pharm. 2017. https://doi.org/10.1016/j.ijpharm.2017. 07.074

[28] T. R. Bhardwaj, M. Kanwar, R. Lal, A. Gupta, Drug Dev. Ind. Pharm. 2000, 26, 1025

[29] R. C. Mundargi, V. R. Babu, V. Rangaswamy, P. Patel, T. M. Aminabhavi, J. Controlled Release 2008, 125, 193.

[30] Y. Qiu, K. Park, Adv. Drug Delivery Rev. 2012, 64, 49.

[31] S. M. Moghimi, A. C. Hunter, J. C. Murray, Pharmacol. Rev. 2001, 53, 283

[32] Y. N. Konan, R. Gurny, E. Allémann, J. Photochem. Photobiol. B Biol. 2002, 66, 89

[33] W. Wang, H. Song, J. Zhang, P. Li, C. Li, C. Wang, D. Kong, Q. Zhao, J. Controlled Release 2015, 203, 57.

[34] Y. Li, J. Rodrigues, H. Tomás, Chem. Soc. Rev. 2012, 41, 2193.

[35] K. K. Bawa, J. K. Oh, Mol. Pharm. 2017, 14, 2460.

[36] A. Bains, Y. Cao, S. Kly, J. E. Wulff, M. G. Moffitt, Mol. Pharm. 2017, 14, 2595.

[37] P. Xiao, J. Zhang, J. Zhao, M. H. Stenzel, Prog. Polym. Sci. 2017.

[38] B. Twaites, C. de las Heras Alarcón, C. Alexander, J. Mater. Chem. 2005, 15, 441.

[39] R. Duncan, M. J. Vicent, Adv. Drug Delivery Rev. 2013, 65, 60.

[40] P. Grossen, D. Witzigmann, S. Sieber, J. Huwyler, J. Controlled Release 2017, 260, 46.

[41] R. Duncan, Nat. Rev. Drug Discovery 2003, 2, 347.

[42] M. C. Branco, J. P. Schneider, Acta Biomater. 2009, 5, 817

[43] M.-H. Li, P. Keller, Soft Matter 2009, 5, 927.

[44] C. E. Mora-Huertas, H. Fessi, A. Elaissari, Int. J. Pharm. 2010, 385 , 113.

[45] C. Alvarez-Lorenzo, A. Concheiro, Chem. Commun. 2014, 50, 7743.

[46] G. Dumortier, J. L. Grossiord, F. Agnely, J. C. Chaumeil, Pharm. Res. 2006, 23, 2709.

[47] A. P. Esser-Kahn, S. A. Odom, N. R. Sottos, S. R. White, J. S. Moore, Macromolecules 2011, 44, 5539.

[48] R. Alvarez-Román, A. Naik, Y. N. Kalia, R. H. Guy, H. Fessi, J. Controlled Release 2004, 99, 53.

[49] C. Leuner, J. Dressman, Eur. J. Pharm. Biopharm. 2000, 50, 47.

[50] J. Breitenbach, Eur. J. Pharm. Biopharm. 2002, 54, 107.

[51] M. E. Gomes, A. S. Ribeiro, P. B. Malafaya, R. L. Reis, A. M. Cunha, Biomaterials 2001, 22, 883 .

[52] K. Unger, P. Salzmann, C. Masciullo, M. Cecchini, G. Koller, A. M. Coclite, ACS Appl. Mater. Interfaces 2017, 9, 17408.

[53] R. K. Bose, S. Nejati, K. K. S. Lau, ECS Trans. 2009, 25, 1229.

[54] K. K. S. Lau, K. K. Gleason, Macromolecules 2006, 39, 3688.

[55] M. Creatore, A. Perrotta, in CVD Polymers: Fabrication of Organic Surfaces and Devices, Wiley-VCH Verlag $\mathrm{GmbH} \&$ Co. KGaA, Weinheim, Germany 2015, p. 111

[56] M. Leskelä, M. Ritala, Thin Solid Films 2002, 409, 138

[57] H. Zhou, S. F. Bent, J. Vac. Sci. Technol. A Vac. Surf. Film 2013, 31, 40801.

[58] J. A. DiMasi, L. Feldman, A. Seckler, A. Wilson, Clin. Pharmacol. Ther. 2010, 87, 272

[59] A. I. Baras, A. S. Baras, K. A. Schulman, Nat. Rev. Drug Discovery 2012, 11, 347.

[60] I. Vroman, L. Tighzert, Materials (Basel) 2009, 2, 307.

[61] L. S. Nair, C. T. Laurencin, Prog. Polym. Sci. 2007, 32, 762.

[62] J. L. Yagüe, A. M. Coclite, C. Petruczok, K. K. Gleason, Macromol. Chem. Phys. 2013, 214, 302.

[63] G. Ozaydin-Ince, A. M. Coclite, K. K. Gleason, Rep. Prog. Phys. 2012 75, 16501.

[64] M. Koenig, R. Kumar, C. Hussal, V. Trouillet, L. Barner, J. Lahann, Macromol. Chem. Phys. 2017, 218, 1600521.
[65] D. D. Burkey, in CVD Polymers: Fabrication of Organic Surfaces and Devices (Ed: K. K. Gleason), Wiley-VCH Verlag GmbH \& Co. KGaA, Weinheim 2015.

[66] K. K. S. Lau, K. K. Gleason, Macromol. Biosci. 2007, 7, 429.

[67] K. Tachibana, M. Nishida, H. Harima, Y. Urano, J. Phys. D. Appl. Phys. 1984, 17, 1727.

[68] H. Yasuda, T. Hirotsu, J. Polym. Sci. Polym. Chem. Ed. 1978, 16, 743.

[69] P. A. Tamirisa, K. C. Liddell, P. D. Pedrow, M. A. Osman, J. Appl. Polym. Sci. 2004, 93, 1317.

[70] S. M. George, Chem. Rev. 2010, 110, 111.

[71] A. A. Dameron, D. Seghete, B. B. Burton, S. D. Davidson, A. S. Cavanagh, J. A. Bertrand, S. M. George, Chem. Mater. 2008, 20, 3315.

[72] A. Tufani, G. Ozaydin-Ince, J. Appl. Polym. Sci. 2015, 132. https:// doi.org/10.1002/app.42453

[73] S. J. P. Mclnnes, E. J. Szili, S. A. Al-Bataineh, J. Xu, M. E. Alf, K. K. Gleason, R. D. Short, N. H. Voelcker, ACS Appl. Mater. Interfaces 2012, 4, 3566

[74] S. J. P. Mclnnes, E. J. Szili, S. A. Al-Bataineh, R. B. Vasani, J. Xu, M. E. Alf, K. K. Gleason, R. D. Short, N. H. Voelcker, Langmuir 2016, $32,301$.

[75] S. H. Baxamusa, K. K. Gleason, Chem. Vap. Deposition 2008, 14, 313.

[76] P. Christian, H. M. A. Ehmann, A. M. Coclite, O. Werzer, ACS Appl. Mater. Interfaces 2016, 8, 21177.

[77] S. Mura, J. Nicolas, P. Couvreur, Nat. Mater. 2013, 12, 991.

[78] P. Christian, H. M. A. Ehmann, O. Werzer, A. M. Coclite, Soft Matter 2016, 12, 9501

[79] P. D. Haller, M. Gupta, Macromol. Rapid Commun. 2014, 35, 2000.

[80] M. E. Alf, A. Asatekin, M. C. Barr, S. H. Baxamusa, H. Chelawat, G. Ozaydin-Ince, C. D. Petruczok, R. Sreenivasan, W. E. Tenhaeff, N. J. Trujillo, S. Vaddiraju, J. Xu, K. K. Gleason, Adv. Mater. 2010, 22, 1993.

[81] M. E. Alf, T. A. Hatton, K. K. Gleason, Thin Solid Films 2011, 519, 4412.

[82] M. E. Alf, T. A. Hatton, K. K. Gleason, Polymer (Guildf) 2011, 52, 4429 .

[83] S. G. Im, K. W. Bong, C.-H. Lee, P. S. Doyle, K. K. Gleason, Lab. Chip 2009, 9, 411 .

[84] J. Xu, K. K. Gleason, Chem. Mater. 2010, 22, 1732

[85] G. Ozaydin-Ince, K. K. Gleason, Chem. Vap. Deposition 2010, 16, 100

[86] P. Moni, A. Al-Obeidi, K. K. Gleason, Beilstein J. Nanotechnol. 2017, 8, 723.

[87] R. B. Vasani, S. J. P. McInnes, M. A. Cole, A. M. M. Jani, A. V. Ellis, N. H. Voelcker, Langmuir 2011, 27, 7843.

[88] X. Huang, C. S. Brazel, J. Controlled Release 2001, 73, 121.

[89] M. A. Ward, T. K. Georgiou, Polymers (Basel) 2011, 3, 1215.

[90] P. Paradiso, V. Chu, L. Santos, A. P. Serro, R. Colaço, B. Saramago, J. Biomed. Mater. Res. Part B Appl. Biomater. 2015, 103, 1059.

[91] C. Susut, R. B. Timmons, Int. J. Pharm. 2005, 288, 253.

[92] S. Osaki, M. Chen, P. O. Zamora, J. Biomater. Sci. Polym. Ed. 2012, 23, 483.

[93] K. Vasilev, N. Poulter, P. Martinek, H. J. Griesser, ACS Appl. Mater. Interfaces 2011, 3, 4831

[94] K. Vasilev, S. S. Griesser, H. J. Griesser, Plasma Process. Polym. 2011, 8, 1010.

[95] S. Zanini, E. Grimoldi, C. Riccardi, Mater. Chem. Phys. 2013, 138, 850.

[96] P. L. Ritger, N. A. Peppas, J. Controlled Release 1987, 5, 23.

[97] P. L. Ritger, N. A. Peppas, J. Controlled Release 1987, 5, 37.

[98] D. P. Dowling, S. Maher, V. J. Law, M. Ardhaoui, C. Stallard, A. Keenan, J. Phys. D. Appl. Phys. 2016, 49, 364005.

[99] S. Bhatt, J. Pulpytel, M. Mirshahi, F. Arefi-Khonsari, Polymer (United Kingdom) 2013, 54, 4820. 
[100] S. Bhatt, F. Valamanesh, J. Pulpytel, R. Lo Dico, A. Baiyukha, I. Al-dybiat, M. Pocard, F. Arefi-Khonsari, M. Mirshahi, Oncotarget 2016, 7, 58121.

[101] C. Labay, J. M. Canal, M. Modic, U. Cvelbar, M. Quiles, M. Armengol, M. A. Arbos, F. J. Gil, C. Canal, Biomaterials 2015, 71, 132.

[102] S. Simovic, D. Losic, K. Vasilev, Chem. Commun. 2010, 46, 1317.

[103] S. J. P. Mclnnes, T. D. Michl, B. Delalat, S. A. Al-Bataineh, B. R. Coad, K. Vasilev, H. J. Griesser, N. H. Voelcker, ACS Appl. Mater. Interfaces 2016, 8, 4467.

[104] C. Canal, K. Khurana, S. Gallinetti, S. Bhatt, J. Pulpytel, F. ArefiKhonsari, M. P. Ginebra, Polymer (United Kingdom) 2016, 92, 170.

[105] C. Labay, J. Buxadera-Palomero, M. Avilés, C. Canal, M. P. Ginebra, J. Phys. D. Appl. Phys. 2016, 49, 304004.

[106] T. O. Kääriäinen, M. Kemell, M. Vehkamäki, M.-L. Kääriäinen, A. Correia, H. A. Santos, L. M. Bimbo, J. Hirvonen, P. Hoppu, S. M. George, D. C. Cameron, M. Ritala, M. Leskelä, Int. J. Pharm. 2017, 525, 160 .

[107] N. A. Vogel, P. S. Williams, A. H. Brozena, D. Sen, S. Atanasov, G. N. Parsons, S. A. Khan, Adv. Mater. Interfaces 2015, 2, 1.

[108] C. Boehler, F. Güder, U. M. Kücükbayrak, M. Zacharias, M. Asplund, Sci. Rep. 2016, 6, 19574.

[109] B. C. Hancock, M. Parks, Pharm. Res. 2000, 17, 397.

[110] C. Leuner, Eur. J. Pharm. Biopharm. 2000, 50, 47.

[111] S. Bosselmann, D. E. Owens, R. L. Kennedy, M. J. Herpin, R. O. Williams, Eur. J. Pharm. Biopharm. 2011, 78, 67.

[112] A. M. Coclite, Y. Shi, K. K. Gleason, Adv. Funct. Mater. 2012, 22, 2167.

[113] P. Zahedi, P. I. Lee, Eur. J. Pharm. Biopharm. 2007, 65, 320.

[114] C. L.-N. Vo, C. Park, B.-J. Lee, Eur.J. Pharm. Biopharm. 2013, 85, 799.

[115] Y. W. Yang, G. Camporeale, E. Sardella, G. Dilecce, J. S. Wu, F. Palumbo, P. Favia, Plasma Process. Polym. 2014, 11, 1102.

[116] F. Palumbo, G. Camporeale, Y. W. Yang, J. S. Wu, E. Sardella, G. Dilecce, C. D. Calvano, L. Quintieri, L. Caputo, F. Baruzzi, P. Favia, Plasma Process. Polym. 2015, 12, 1302.

[117] C. Hsiao, C.-C. Wu, Y. Liu, Y. Yang, Y. Cheng, F. Palumbo, G. Camporeale, P. Favia, J. Wu, IEEE Trans. Plasma Sci. 2016, 44, 3091.

[118] M. J. Garcia-Fernandez, L. Martinez-Calvo, J.-C. Ruiz, M. R. Wertheimer, A. Concheiro, C. Alvarez-Lorenzo, Plasma Process. Polym. 2012, 9, 540.

[119] C. Lo Porto, F. Palumbo, G. Palazzo, P. Favia, Polym. Chem. 2017, 8 , 1746.

[120] S.-W. Myung, S.-C. Jung, B.-H. Kim, Thin Solid Films 2015, 584, 13.

[121] S.-J. Song, Y. J. Park, J. Park, M. D. Cho, J.-H. Kim, M. H. Jeong, Y. S. Kim, D. L. Cho, J. Mater. Chem. 2010, 20, 4792

[122] C. Canal, M. Modic, U. Cvelbar, M.-P. Ginebra, Biomater. Sci. 2016 4, 1454.

[123] Y. Yamauchi, M. Kuzuya, Y. Sasai, S. Kondo, J. Photopolym. Sci. Technol. 2013, 26, 529.
[124] Y. Yamauchi, M. Kuzuya, Y. Sasai, S. Kondo, J. Photopolym. Sci. Technol. 2014, 27, 389.

[125] Y. Yamauchi, M. Kuzuya, Y. Sasai, J. Photopolym. Sci. Technol. 2016, 29, 447.

[126] K. Hagiwara, T. Hasebe, A. Hotta, Surf. Coat. Technol. 2013, 216, 318.

[127] R. Langer, Nature 1998, 392, 5.

[128] M. E. Alf, P. D. Godfrin, T. A. Hatton, K. K. Gleason, Macromol. Rapid Commun. 2010, 31, 2166.

[129] A. C. Pauly, K. Schöller, L. Baumann, R. M. Rossi, K. Dustmann, U. Ziener, D. de Courten, M. Wolf, L. F. Boesel, L. J. Scherer, Sci. Technol. Adv. Mater. 2015, 16, 34604.

[130] A. Gupta, V. K. Patel, R. Kant, S. Bhattacharya, Rev. Adhes. Adhes. 2016, 4, 166.

[131] G. Ozaydin-Ince, K. K. Gleason, M. C. Demirel, Soft Matter 2011, 7, 638.

[132] B. Ziaie, A. Baldi, M. Lei, Y. Gu, R. A. Siegel, Adv. Drug Delivery Rev. 2004, 56, 145.

[133] A. Baldi, Y. D. Gu, P. E. Loftness, R. A. Siegel, B. Ziaie, J. Microelectromech. Syst. 2003, 12, 613.

[134] L. He, S. Sarkar, A. Barras, R. Boukherroub, S. Szunerits D. Mandler, Chem. Commun. 2017, 53, 4022.

[135] A. Jonsson, T. A. Sjöström, K. Tybrandt, M. Berggren, D. T. Simon, Sci. Adv. 2016, 2, e 1601340.

[136] I. Lee, J. He, M. Tsai, K. Lin, J. Mater. Chem. B Mater. Biol. Med. 2014, 3, 276.

[137] K. Nair, B. Whiteside, C. Grant, R. Patel, C. Tuinea-Bobe, K. Norris, A. Paradkar, Pharmaceutics 2015, 7, 471 .

[138] S. M. George, B. Yoon, A. A. Dameron, Acc. Chem. Res. 2009, 42, 498.

[139] P. Sundberg, M. Karppinen, Beilstein J. Nanotechnol. 2014, 5, 1104

[140] C. Hossbach, F. Nehm, A. Singh, H. Klumbies, D. Fischer, C. Richter, U. Schroeder, M. Albert, L. Müller-Meskamp, K. Leo, T. Mikolajick, J. W. Bartha, J. Vac. Sci. Technol. A Vac. Surf. Film 2015 33, $01 \mathrm{~A} 119$

[141] S. Bent, P. W. Loscutoff, S. Clendenning, MRS Proc. 2010, 1249, 1249.

[142] X. Liang, A. W. Weimer, J. Nanopart. Res. 2010, 12, 135.

[143] T. D. Gould, A. Izar, A. W. Weimer, J. L. Falconer, J. W. Medlin, ACS Catal. 2014, 4, 2714.

[144] Y. Chen, B. Zhang, Z. Gao, C. Chen, S. Zhao, Y. Qin, Carbon N. Y. 2015, 82, 470

[145] B. Jeong, Y. H. Bae, D. S. Lee, Nature 1997, 388, 860.

[146] X. Dong, C. Wei, T. Liu, F. Lv, Z. Qian, ACS Appl. Mater. Interfaces 2016, 8, 5104

[147] G. Ozaydin-Ince, J. M. Dubach, K. K. Gleason, H. A. Clark, PNAS 2011, 108, 2656.

[148] P. Poodt, D. C. Cameron, E. Dickey, S. M. George, V. Kuznetsov G. N. Parsons, F. Roozeboom, G. Sundaram, A. Vermeer, J. Vac. Sci. Technol. A Vac. Surf. Film 2012, 30, 10802. 